\title{
TRANSFINITE GRAPHS AND ELECTRICAL NETWORKS
}

\author{
A. H. ZEMANIAN
}

\begin{abstract}
All prior theories of infinite electrical networks assume that such networks are finitely connected, that is, between any two nodes of the network there is a finite path. This work establishes a theory for transfinite electrical networks wherein some nodes are not connected by finite paths but are connected by transfinite paths. Moreover, the voltages at those nodes may influence each other. The main difficulty to surmount for this extension is the construction of an appropriate generalization of the concept of connectedness. This is accomplished by extending the idea of a node to encompass infinite extremities of a graph. The construction appears to be novel and leads to a hierarchy of transfinite graphs indexed by the finite and infinite ordinals. Two equivalent existence and uniqueness theorems are established for transfinite resistive electrical networks based upon Tellegen's equation, one using currents and the other using voltages as the fundamental quantities. Kirchhoff's laws do not suffice for this purpose and indeed need not hold everywhere in infinite networks. Although transfinite countable electrical networks have in general an uncountable infinity of extremities, called "tips," the number of different tip voltages may be radically constrained by both the graph of the network and its resistance values. Conditions are established herein under which various tip voltages are compelled to be the same. Furthermore, a theorem of Shannon-Hagelbarger on the concavity of resistance functions is extended to the driving-point resistance between any two extremities of arbitrary ranks. This is based upon an extension of Thomson's least power principle to transfinite networks.
\end{abstract}

\section{INTRODUCTION}

This paper is divided into two parts. The first part $(\S \S 2-7)$ generalizes the idea of connectedness to allow infinite graphs to be joined together at their infinite extremities to obtain transfinite graphs. Such joining occurs at "1-nodes" and is analogous to the incidence of branches at nodes (henceforth called " 0 -nodes"). The key idea [21, 24] is the following: One can "arrive at infinity" through an infinite graph by tracing a one-ended path (i.e., a one-way infinite path). However, the "extremity of the graph" that is thereby reached should be defined independently of the path that is chosen. This is accomplished by defining that extremity as an equivalence class of one-ended paths, every two paths of which

Received by the editors September 4, 1989 and, in revised form, August 13, 1990.

1980 Mathematics Subject Classification (1985 Revision). Primary 05C99, 94C05; Secondary $05 \mathrm{C} 40,94 \mathrm{C} 15$.

Key words and phrases. Infinite electrical networks, transfinite graphs, transfinite connectedness, currents at and beyond infinity.

Research on this paper was supported by the National Science Foundation under Grants DMS8521824 and MIP-8822774. 
differ by no more than a finite number of branches. That equivalence class is called a "0-tip". Then, a "1-node" is a specified set of 0 -tips and is understood to "connect" those 0-tips together. The result is a transfinite graph, called a "1-graph". In a similar way, equivalence classes of one-ended transfinite paths in 1-graphs define the extremities of the 1-graph, called "1-tips", and these can be connected together through "2-nodes" to get "2-graphs". In fact, the process can be repeated to obtain " $k$-graphs", where $k$ may be a natural number or a transfinite ordinal; this yields a hierarchy of transfinite graphs indexed by $k$.

The purpose of this generalization of graph theory is to create a structure upon which can be based a theory for infinite resistive electrical networks that allows the flow of current out to infinity and beyond. Section 8 presents a simple example demonstrating the need for such an extension of electrical network theory; that section also summarizes the primary results of $\S \S 9-17$, which are devoted to a most general theory for transfinite electrical networks. Furthermore, this structure empowers the idea of a transfinite random walk, a generalization of a random walk on an ordinary infinite graph, wherein the walker may "wander through infinity" by passing through a " $k$ node", where $k$ can be any transfinite countable ordinal [28, 29].

Just a few remarks about terminology: As usual, a partition $\left\{x_{m}\right\}$ of a nonvoid set $y$ means that $y=\bigcup x_{m}$, each $x_{m}$ is nonvoid, and $x_{m} \cap x_{n}$ is void for $m \neq n$. A singleton is a set with exactly one member. A generic form of a phrase we shall often use is " $k$-entity", where $k$ is an ordinal and in place of "entity" we will have "tip", "node", "path", "section", or "network"; we shall refer to $k$ as the rank of the entity.

The terminology of this paper does not conform to that of the prior works $[21,24]$, which were precursors for this one. Those prior works were concerned with connections "at infinity" whereas this one goes "beyond infinity". An attempt to maintain that prior terminology leads to an unnecessarily complicated nomenclature.

Finally, the anonymous Referee's help in improving the mathematical style of this paper needs to be acknowledged. I am grateful for his suggestions-and patience.

\section{PART 1 \\ 2. 0-GRAPHS}

A 0-graph is a conventional countable graph consisting of a countable set $\mathscr{B}$ of branches, a countable set $\mathscr{N}^{0}$ of nodes (henceforth called 0-nodes), and a mapping from $\mathscr{B}$ into the set of all single-element and two-element subsets of $\mathscr{N}^{0}$. That mapping defines the incidence between branches and nodes. If a particular branch is thereby mapped into a single-element subset of $\mathscr{N}^{0}$, it is called a selfloop. A 0 -graph is denoted by $\mathscr{G}^{0}=\left(\mathscr{B}, \mathscr{N}^{0}\right)$, the said mapping being understood.

Our terminology concerning 0-graphs is conventional except for the following modifications: Adjectives and nouns such as adjacent, subgraph, finite 0-node, and infinite 0 -node have their usual meanings. Other standard phrases will have a "0-" appended as prefixes to distinguish them from analogous concepts of higher ranks, which will be introduced in later sections. Thus, we speak of 0-paths, 0-loops, and 0-connectedness. The phrase finitely connected means 0- 
connected, that is, connected in the usual sense. If a 0-path is one-way infinite or two-way infinite, it is called one-ended or respectively endless. A 0-path is called nontrivial if it has at least one branch. A trivial 0 -path consists of just one 0 -node. A 0-loop is a finite nontrivial 0-path except that it closes on itself, the first and last elements being the same 0-node. Disjoint 0-paths and 0-loops will be called totally disjoint in order to conform with some subsequent terminology. For the same reason, a 0 -node is said to embrace itself, and a 0-path is said to embrace itself as well as all its nodes and branches.

A 0 -section of a 0 -graph is a maximal subgraph that is 0 -connected. At this early stage of our definitions, a 0 -section is simply a component of the 0 -graph in the usual sense, but we will shortly generalize the idea of connectedness and thereby render a 0 -section into something other than a component.

\section{1-GRAPHS}

Definition (0-tip). Two one-ended 0-paths in a 0-graph $\mathscr{G}^{0}=\left(\mathscr{B}, \mathscr{N}^{0}\right)$ are taken to be equivalent if they are identical except for a finite number of nodes and branches. This equivalence relationship partitions the set of all one-ended 0 -paths in $\mathscr{G}^{0}$ into equivalence classes, which will be called the 0-tips of $\mathscr{G}^{0}$. (These were called "pathlike extremities" in [24].) A representative of a 0-tip is any one-ended path in that equivalence class.

This idea of 0-tips is fundamental to our discussion, for it is to them that "connections at infinity" will be made. Although the branch set $\mathscr{B}$ is countable, $\mathscr{G}^{0}$ may have an uncountably infinite set of 0 -tips. For example, this is the case when $\mathscr{G}^{0}$ is the infinite binary tree.

Definition (possession of a 0 -tip). If a representative of a 0 -tip $t^{0}$ is a subgraph of a subgraph $\mathscr{G}_{s}^{0}$ of a 0 -graph $\mathscr{G}^{0}$, then $\mathscr{G}_{s}^{0}$ is said to have, or possess $t^{0}$.

Note that an infinite subgraph $\mathscr{G}_{s}^{0}$ or even $\mathscr{G}^{0}$ itself may not possess any 0 -tips because it may not contain any one-ended paths. In the event that $\mathscr{G}^{0}$ does possess 0 -tips, we can extend the idea of a node to include the "infinite extremities" of a 0 -graph as follows: First, partition the set $\mathscr{T}^{0}$ of all 0 tips into subsets $\mathscr{T}_{\tau}^{0}$; thus, $\mathscr{T}^{0}=\bigcup \mathscr{T}_{\tau}^{0}$, where $\tau$ denotes the indices of the partition. Each $\mathscr{T}_{\tau}^{0}$ may be either finite, denumerable, or uncountably infinite, but it is not void. Secondly, for each $\tau$ let $\mathscr{N}_{\tau}^{0}$ be either the void set or a singleton whose element is a 0 -node; furthermore, we require that $\mathscr{N}_{\tau_{1}}^{0} \cap \mathcal{N}_{\tau_{2}}^{0}=\varnothing$ if $\tau_{1} \neq \tau_{2}$.

Definition (1-node). For each $\tau$ the set $\mathscr{T}_{\tau}^{0} \cup \mathscr{N}_{\tau}^{0}$ is called a 1-node.

In the event that $\mathscr{T}_{\tau}^{0}$ is a countable set, the corresponding 1 -node $x^{1}$ may be written out as

$$
x^{1}=\left\{x_{0}^{0}, t_{1}^{0}, t_{2}^{0}, t_{3}^{0}, \ldots\right\},
$$

where the $t_{m}^{0}$ are 0 -tips and $x_{0}^{0}$ is a 0 -node, which may not be present. Our definition insures that every 1 -node contains at least one 0 -tip and every 0 -tip appears in one and only one 1-node. Also, every 1 -node contains at most one 0 node and perhaps none at all, and no 0 -node appears in more than one 1-node; in fact, a particular 0 -node may not be a member of any 1-node. 
Definition (exceptional element). If $\mathcal{N}_{\tau}^{0}$ is not void, the 0 -node in $\mathscr{N}_{\tau}^{0}$ is called the exceptional element of the 1 -node $\mathscr{N}_{\tau}^{0} \cup \mathscr{T}_{\tau}^{0}$.

A physical interpretation of a 1-node is that of a short circuit connected to its elements. This will allow the flow of current along a path out to infinity, through a short circuit at infinity, and then along another infinite path. Alternatively, the current may jump along a short circuit from a node out to infinity and then continue along an infinite path.

Definition (to embrace-for 1-nodes). A 1-node is said to embrace itself as well as all its elements, that is, its 0-tips and its exceptional element, if that exists. However, we take it that it does not embrace the representatives of its 0 -tips, nor any other entity.

We will use some natural vocabulary in discussing these concepts; in order to be precise, let us explain that vocabulary. Assume that $x_{0}^{0}$ is the exceptional element of a 1-node $x^{1}$. A branch that is incident to $x_{0}^{0}$ is said to meet $x^{1}$. A 0 -path $P^{0}$ that contains $x_{0}^{0}$ is said to meet $x^{1}$ at $x_{0}^{0}$, and, if $x_{0}^{0}$ is a terminal node of $P^{0}$, then $P^{0}$ is said to terminate at $x^{1}$ with $x_{0}^{0}$. Similarly, a oneended or endless 0 -path that contains a representative of a 0 -tip $t^{0}$ in $x^{1}$ is said to meet $x^{1}$ with $t^{0}$.

Definition (1-graph). A 1-graph is a triplet $\mathscr{G}^{1}=\left(\mathscr{B}, \mathscr{N}^{0}, \mathscr{N}^{1}\right)$, where $\mathscr{B}$ and $\mathscr{N}^{0}$ denote the branch set and 0 -node set of a 0 -graph and $\mathscr{N}^{1}$ is a specified set of 1 -nodes constructed from the 0 -tips and 0 -nodes of that 0 -graph. It is required that $\mathscr{N}^{1}$ be nonvoid; otherwise, the 1-graph is taken to be nonexistent.

Note that the possibility of a void $\mathscr{N}^{1}$ exists because $\mathscr{G}$ may not have any one-ended paths. Also, to identify $\mathscr{N}^{1}$, we need merely specify the 1 -nodes that are not singletons.

Example 3.1. As an example, consider Figure 1, which shows an infinite lattice cascade $\mathscr{G}_{1}$ and an infinite ladder $\mathscr{G}_{2}$ that are "connected at infinity" in such a fashion that their infinite extensions reach toward each other. The line segments indicate branches, the heavy dots indicate 0-nodes, and the two small circles

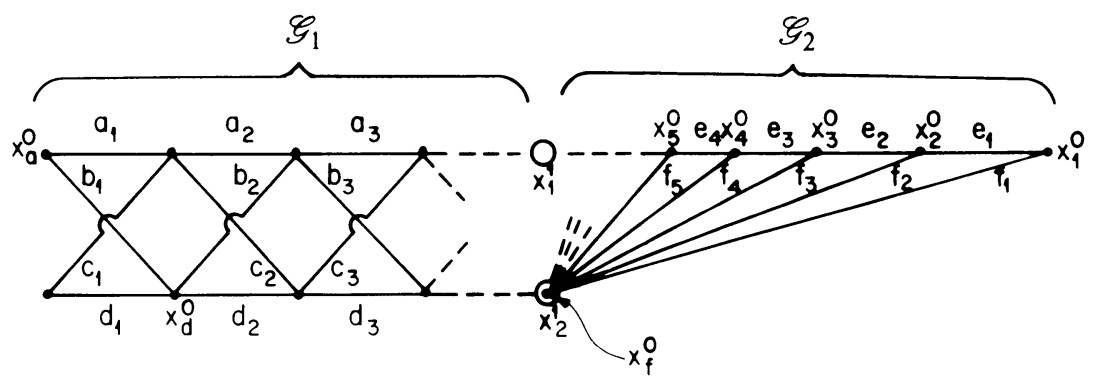

FiguRE 1. $\mathscr{G}_{1}$ is an infinite cascade of lattices connected at infinity to an infinite ladder $\mathscr{G}_{2}$. Each labeled line segment denotes a branch. $x_{1}^{1}$ and $x_{2}^{1}$ are 1-nodes; $x_{2}^{1}$ embraces the infinite 0 -node $x_{f}^{0}$ of the ladder network. 
indicate 1-nodes, which will be specified momentarily. We can identify any 0 path just by listing its branches in sequence. For instance, we have the following 0 -paths.

$$
\begin{aligned}
P_{a} & =\left\{a_{1}, a_{2}, a_{3}, \ldots\right\}, \\
P_{b} & =\left\{b_{1}, b_{2}, b_{3}, \ldots\right\}, \\
P_{a b c} & =\left\{a_{1}, c_{2}, c_{3}, a_{4}, b_{5}, b_{6}, a_{7}, \ldots\right\}, \\
P_{d} & =\left\{d_{2}, d_{3}, d_{4}, \ldots\right\}, \\
P_{e} & =\left\{e_{1}, e_{2}, e_{3}, \ldots\right\} .
\end{aligned}
$$

$\mathscr{G}_{1}$ has an infinity of 0-tips. Representatives of three of them are $P_{a}, P_{b}$, and $P_{a b c}$ respectively. Even though $P_{a}, P_{b}$, and $P_{a b c}$ have an infinity of nodes in common, they represent distinct 0-tips, which need not be declared to be "connected at infinity", that is, members of the same 1-node.

Let $t_{a}, t_{d}$, and $t_{e}$ be the 0-tips with the representatives $P_{a}, P_{d}$, and $P_{e}$ respectively. Also, let $x_{f}^{0}$ be the infinite node of the ladder network $\mathscr{G}_{2}$. We might take as our nonsingleton 1-nodes the two sets $x_{1}^{1}=\left\{t_{a}, t_{e}\right\}$ and $x_{2}^{1}=$ $\left\{x_{f}^{0}, t_{d}\right\}$. This is what is intended in Figure 1. According to some of our forthcoming definitions, this allows the flow of a "1-loop current" along $P_{a}$, through $x_{1}^{1}$, along $P_{e}$ in the reverse direction, along branch $f_{1}$, through $x_{2}^{1}$, along $P_{d}$ in the reverse direction, and finally along branch $b_{1}$. Moreover, we have at hand the 1-graph $\left(\mathscr{B}, \mathscr{N}^{0}, \mathscr{N}^{1}\right)$ where $\mathscr{B}$ and $\mathscr{N}^{0}$ are implicitly specified by $\mathscr{G}_{1}$ and $\mathscr{G}_{2}$ together.

Alternatively, we could construct another 1-graph by letting $\mathscr{N}^{1}$ have only one nonsingular 1-node, namely, $x^{1}=\left\{t_{a}, t_{b}\right\}$, where $t_{a}$ is as before and $t_{b}$ is the 0-tip with $P_{b}$ as a representative. This would disconnect $\mathscr{G}_{1}$ from $\mathscr{G}_{2}$. On the other hand, it would allow the flow of current along $P_{a}$, through $x_{1}$, and backwards along $P_{b}$. This flow would not be a 1-loop current according to our upcoming definition because $P_{a}$ and $P_{b}$ share nodes.

Let $\mathscr{B}_{r}$ be a nonvoid subset of $\mathscr{B}$ and let $\left(\mathscr{B}_{r}, \mathscr{N}_{r}^{0}\right)$ be the subgraph of the 0 -graph $\left(\mathscr{B}, \mathscr{N}^{0}\right)$ induced by $\mathscr{B}_{r}$. Corresponding to every 0-tip of $\left(\mathscr{B}_{r}, \mathscr{N}_{r}^{0}\right)$ there is a unique 0 -tip of $\left(\mathscr{B}, \mathscr{N}^{0}\right)$ containing the representatives of the first 0 -tip. However, there may be 0 -tips in $\left(\mathscr{B}, \mathscr{N}^{0}\right)$ which do not exist as 0 -tips in $\left(\mathscr{B}_{r}, \mathscr{N}_{r}^{0}\right)$ because $\left(\mathscr{B}_{r}, \mathscr{N}_{r}^{0}\right)$ does not contain any representatives of those 0 tips. Now, let $x^{1}$ be any 1-node for the 1-graph $\mathscr{G}^{1}=\left(\mathscr{B}, \mathscr{N}^{0}, \mathscr{N}^{1}\right)$. Remove every 0-tip in $x^{1}$ that does not exist as a 0 -tip in $\left(\mathscr{B}_{r}, \mathscr{N}_{r}^{0}\right)$. The resulting set $x_{r}^{1}$ is called a reduced 1-node (induced by $\mathscr{B}_{r}$ ) if it possesses at least one 0tip-and even if no 0-tips were removed. Let $\mathscr{N}_{r}^{1}$ be the set of all reduced 1-nodes. If $\mathscr{N}_{r}^{1}$ is not void, let $\mathscr{G}_{r}$ be the 1-graph $\left(\mathscr{B}_{r}, \mathscr{N}_{r}^{0}, \mathscr{N}_{r}^{1}\right)$; if $\mathscr{N}_{r}^{1}$ is void, let $\mathscr{G}_{r}$ be the 0 -graph $\left(\mathscr{B}_{r}, \mathscr{N}_{r}^{0}\right)$.

Definition (reduced graph). $\mathscr{G}_{r}$ is called the reduction of $\mathscr{G}^{1}$ with respect to $\mathscr{B}_{r}$ or the reduced graph induced by $\mathscr{B}_{r}$.

The idea of 0 -connectedness applies to any 1-graph $\left(\mathscr{B}, \mathscr{N}^{0}, \mathscr{N}^{1}\right)$ since 0 paths are defined in terms of $\mathscr{B}$ and $\mathscr{N}^{0}$. For instance, the subgraph $\mathscr{G}_{1}$ of Figure 1 is 0 -connected (in fact, is a 0 -section) but the entire graph $\mathscr{G}$ is not because there is no finite 0 -path that connects a node of $\mathscr{G}_{1}$ to a node of $\mathscr{G}_{2}$. 
However, by generalizing the idea of a path, we can say that $\mathscr{G}$ is connected in a wider sense.

We need some more definitions. Let $x$ be either a 0 -node or a 1 -node, and similarly for $y$. Also, let $P^{0}$ be a 0-path.

Definition (totally disjoint-for 0 -nodes and 1-nodes). $x$ and $y$ are said to be totally disjoint if they do not embrace a common element. Also, $x$ and $P^{0}$ are called totally disjoint if $P^{0}$ does not meet $x$.

Definition (terminally incident). $x$ and $P^{0}$ are said to be terminally incident if $P^{0}$ meets $x$ either at a terminal node or with a 0 -tip. In this case, $x$ and $P^{0}$ are said to be terminally incident but otherwise totally disjoint if they do not meet at any other node or with another 0 -tip of $P^{0}$; thus, $P^{0}$ meets $x$ with only one (not both) of its ends or tips.

Definition (1-path). The alternating sequence

$$
\left\{\ldots, x_{m}^{1}, P_{m}^{0}, x_{m+1}^{1}, P_{m+1}^{0}, \ldots\right\}
$$

is called a 1-path if the following three conditions are fulfilled.

(i) Each $P_{m}^{0}$ is a nontrivial 0-path and each $x_{m}^{1}$ is a 1-node except possibly when (1) terminates on the left and/or on the right; in the latter case, each terminal element is either a 0 -node or a 1 -node.

(ii) Each $P_{m}^{0}$ is terminally incident to the two nodes immediately preceding and succeeding it in (1) but is otherwise totally disjoint from those nodes.

(iii) Every two elements in (1) that are not adjacent therein are totally disjoint.

By designating (1) as a sequence, we understand that (1) is a totally ordered set whose elements can be numbered consecutively by an index $m$ that is restricted to some or all of the integers. This is an essential distinction, for later on $(\S 7)$ we will discuss other kinds of paths whose elements cannot be so numbered-as, for example, when $m$ extends into the transfinite ordinals.

A 1-path is called nontrivial if it has at least three elements. The adjectives, finite, one-ended, and endless, are defined for 1-paths just as they are for 0paths.

Definition (1-loop). A 1-loop is a finite 1-path except for the following requirement: One of the two terminal elements embraces the other.

Let $P_{1}^{1}$ and $P_{2}^{1}$ each denote either a 1-path or a 1-loop.

Definition (totally disjoint). $P_{1}^{1}$ and $P_{2}^{1}$ are said to be totally disjoint if every node or 0-path in $P_{1}^{1}$ is totally disjoint from every node and every 0-path in $P_{2}^{1}$.

Example 3.2. Refer to Figure 1 again. We use the same notation as before. Also, the $x$ 's denote nodes as indicated. Then,

$$
P_{e f}=\left\{\ldots, e_{3}, x_{3}^{0}, e_{2}, x_{2}^{0}, e_{1}, x_{1}^{0}, f_{1}, x_{f}^{0}\right\}
$$

is a one-ended 0 -path, and

$$
P_{a e f}^{1}=\left\{x_{a}^{0}, P_{a}, x_{1}^{1}, P_{e f}, x_{2}^{1}\right\}
$$

is a finite 1-path. The 0-path $P_{d}$ is terminally incident to $x_{2}^{1}$ but is otherwise totally disjoint from $x_{2}^{1}$. Also, $\left\{x_{a}^{0}, P_{a}, x_{1}^{1}\right\}$ and $\left\{x_{d}^{0}, P_{d}, x_{2}^{1}\right\}$ are totally 
disjoint 1-paths, but $\left\{x_{d}^{0}, P_{d}, x_{2}^{1}\right\}$ and $\left\{x_{1}^{1}, P_{e f}, x_{f}^{0}\right\}$ are not because $x_{2}^{1}$ embraces $x_{f}^{0}$.

Definition (1-connected). Let $x_{a}$ be a 0 -node or a 1-node, and similarly for $x_{b}$. Then, $x_{a}$ and $x_{b}$ are said to be 1-connected if there exists a finite 1-path with $x_{a}$ and $x_{b}$ as its terminal elements. (This meaning for "1-connected" is different from the customary one in conventional graph theory.) Two branches are said to be 1-connected if their 0-nodes are 1-connected. A 1-graph is said to be 1-connected if every two branches are 1-connected.

It follows directly from our definitions that, if two nodes $x_{a}$ and $x_{b}$ are 0 -connected, then they are also 1-connected. Indeed, let $P^{0}$ be a finite 0 -path with $x_{a}$ and $x_{b}$ as its terminal nodes. Then, $\left\{x_{a}, P^{0}, x_{b}\right\}$ is a finite 1-path.

Definition (1-section). A 1-section of a 1-graph $\mathscr{G}$ is a reduction of $\mathscr{G}$ induced by a maximal set of branches that are pairwise 1-connected.

For instance, the graph $\mathscr{G}$ of Figure 1 is 1 -connected but not 0 -connected and is a 1 -section by itself. On the other hand, $\mathscr{G}_{1}$ and $\mathscr{G}_{2}$ are 0 -sections but, under our generalized concept of connectedness, are not components (i.e., disconnected parts) of $\mathscr{G}$. Neither $\mathscr{G}_{1}$ nor $\mathscr{G}_{2}$ is a 1 -section because it is not maximal with respect to 1-connectedness.

A 1-path cannot proceed from one 0 -section to another 0 -section without passing through a 1-node. It may enter or leave a 0 -section either through a 0 -node or through a 0 -tip, and its sojourn within that section may be either a finite, one-ended, or endless 0-path.

\section{4. $p$-GRAPHS}

We now apply recursion to the definitions given in $\S \S 2$ and 3 . Let $p$ be a natural number greater than 1 . Assume that for each $q=0,1, \ldots, p-1$ the $q$-graphs $\left(\mathscr{B}, \mathscr{N}^{0}, \ldots, \mathscr{N}^{q}\right)$ have been defined for a given branch set $\mathscr{B}$ and specified sets $\mathscr{N}^{q}$ of $q$-nodes, and also defined are the $q$-paths $P^{q}, q$ connectedness, and $q$-sections, along with the terminology pertaining to these ideas. This has explicitly been done for $q=0$ and $q=1$, and the constructions of this section will extend those definitions by induction to every natural number $p$.

We start with the $(p-1)$-graph $\mathscr{G}^{p-1}=\left(\mathscr{B}, \mathscr{N}^{0}, \ldots, \mathscr{N}^{p-1}\right)$.

Definition $((p-1)$-tips). Two one-ended $(p-1)$-paths are called equivalent if they differ at most by a finite number of $(p-1)$-nodes and $(p-2)$-paths. This equivalence relationship partitions the set of all one-ended $(p-1)$-paths in $\mathscr{G}^{p-1}$ into equivalence classes, called $(p-1)$-tips. A representative of a $(p-1)$-tip is any one of its members.

$\mathscr{G}^{p-1}$ need not contain any one-ended $(p-1)$-paths and therefore any $(p-1)$ tips, but in the event that it does, we may partition the set $\mathscr{T}^{p-1}$ of all $(p-1)$ tips into subsets $\mathscr{T}_{\tau}^{p-1}$ to get $\mathscr{T}^{p-1}=\bigcup \mathscr{T}_{\tau}^{p-1}$, where again $\tau$ denotes the index of a subset. No $\mathscr{T}_{\tau}^{p-1}$ is void. Furthermore, for each $\tau$ let $\mathscr{N}_{\tau}^{p-1}$ be either the void set or a singleton whose element is a $q$-node, where $0 \leq q \leq$ $p-1$. 
Definition ( $p$-node). For each $\tau$, the set

$$
x_{\tau}^{p}=\mathscr{T}_{\tau}^{p-1} \cup \mathscr{N}_{\tau}^{p-1}
$$

is called a $p$-node.

Definition (exceptional element). The element of $\mathscr{N}_{\tau}^{p-1}$, if it exists, is called the exceptional element of $x_{\tau}^{p}$.

We impose one more condition, which the exceptional elements, taken together, are required to satisfy.

Condition $\Gamma^{p}$. If a p-node contains a q-node $(q<p)$ as an exceptional element, then that $q$-node does not appear as the exceptional element of any other $m$-node, where $q<m \leq p$.

Definition (to embrace-for $p$-nodes). A $p$-node $x^{p}$ is said to embrace itself, and all its elements, and all the elements of its exceptional element $x^{q}$ if it has one, and all the elements of the exceptional element of $x^{q}$ if that exists, and so forth for the exceptional elements of decreasing ranks that arise in this way. However, we take it that a $p$-node does not embrace any other entity; in particular, it does not embrace the representatives of its tips nor the representatives of the tips in the aforementioned exceptional elements. If $x_{1}^{q}$ and $x_{2}^{n}$ are nodes of ranks $q$ and $n$ respectively, where $n \leq q \leq p, x_{1}^{q}$ is said to embrace $x_{2}^{n}$ if $x_{1}^{q}$ embraces all the elements embraced by $x_{2}^{n}$ including $x_{2}^{n}$ itself.

Definition (totally disjoint nodes). The $q$-node $x_{1}^{q}$ and the $m$-node $x_{2}^{m}$ are called totally disjoint if their sets of embraced elements have a void intersection.

Proposition 4.1. If $x_{0}^{q}$ and $y_{0}^{p}$ are respectively $a$-node and a p-node with $0 \leq q \leq p$ and if $x_{0}^{q}$ and $y_{0}^{p}$ embrace a common node, then $y_{0}^{p}$ embraces $x_{0}^{q}$. If in addition $p=q$, then $x_{0}^{p}=y_{0}^{p}$.

Proof. Let $z^{n}$ denote an $n$-node that is embraced by both $x_{0}^{q}$ and $y_{0}^{p}$. If $n=q$, then, since by definition $x_{0}^{q}$ does not embrace another node of the same rank $q$ but does embrace itself, we must have that $z^{n}=x_{0}^{q}$, and so $y_{0}^{p}$ embraces $z^{n}=x_{0}^{q}$.

Now assume that $n<q \leq p$. Let $x_{-1}$ be the unique exceptional element in $x_{0}^{q}$, and let $x_{-k}$ be the unique exceptional element in $x_{-k+1}$ for $k=$ $2,3, \ldots$, . Thus, we have a finite sequence of nodes $x_{-1}, x_{-2}, \ldots$ of strictly decreasing ranks, one of which is the $n$-node $z^{n}$. Similarly, let $y_{-1}, y_{-2}, \ldots$ comprise the sequence of unique exceptional elements of strictly decreasing ranks such that $\cdots \in y_{-2} \in y_{-1} \in y_{0}^{p} ; z^{n}$ is also one of those elements. Suppose that $y_{0}^{p}$ does not embrace $x_{0}^{q}$. It follows that there will be a node $w=x_{-i}=y_{-j}$ appearing in both sequences such that its predecessors $x_{-i+1}$ and $y_{-j+1}(i, j \geq 1)$ are not the same. This violates Condition $\Gamma^{p}$. We can conclude that $y_{0}^{p}$ embraces $x_{0}^{q}$.

If $q=p$, we must have that $x_{0}^{q}=y_{0}^{p}$ because again a $p$-node cannot embrace another $p$-node.

Definition ( $p$-graph). A $p$-graph is a $(p+2)$-tuplet

$$
\mathscr{G}^{p}=\left(\mathscr{B}, \mathscr{N}^{0}, \ldots, \mathscr{N}^{p}\right),
$$


where $\mathscr{B}$ is a set of branches and each $\mathscr{N}^{q}$ for $q=0, \ldots, p$ is a specified nonvoid set of $q$-nodes.

For each $q$ the specification of $\mathscr{N}^{q}$ occurs when the partition $\bigcup \mathscr{T}_{\tau}^{q-1}$ and the $\mathscr{N}_{\tau}^{q-1}$ are chosen. This can be done only after the $\mathscr{N}^{m}$ for $m=0, \ldots$, $q-1$ have been specified. As we shall see, in order for $\mathscr{N}^{p}$ to be nonvoid, the $\mathscr{N}^{q}$, where $q=0, \ldots, p-1$, must be infinite sets.

Given $\mathscr{G}^{p}$ with $p>1$, we can examine the corresponding $\mathscr{G}^{p-1}=\left(\mathscr{B}, \mathscr{N}^{0}\right.$, $\left.\ldots, \mathscr{N}^{p-1}\right)$. Once again, let $\mathscr{B}_{r}$ be a subset of $\mathscr{B}$. Let $\mathscr{G}_{r}^{p-1}=\left(\mathscr{B}_{r}, \mathscr{N}_{r}^{0}\right.$, $\ldots, \mathscr{N}_{r}^{p-1}$ ) be the reduction of $\mathscr{G}^{p-1}$ induced by $\mathscr{B}_{r}$. (This has been explicitly defined for $p-1=1$.) We assume for now that $\mathscr{N}_{r}^{0}, \ldots, \mathscr{N}_{r}^{p-1}$ are all nonvoid. Corresponding to each $(p-1)$-tip $t^{p-1}$ of $\mathscr{G}_{r}^{p-1}$ there is a unique $(p-1)$-tip of $\mathscr{G}^{p-1}$, namely, the one containing the representatives of $t^{p-1}$. However, there may be $(p-1)$-tips in $\mathscr{G}^{p-1}$ that do not exist in $\mathscr{G}_{r}^{p-1}$ because $\mathscr{G}_{r}^{p-1}$ may not contain any representatives of those $(p-1)$-tips. Let $x^{p}$ be any $p$-node of the given $p$-graph $\mathscr{G}^{p}$. Remove every $(p-1)$-tip in $x^{p}$ that does not exist as a $(p-1)$-tip of $\mathscr{G}_{r}^{p-1}$. Similarly, if $x^{p}$ has an exceptional element $x_{0}^{q}$, where $0 \leq q \leq p-1$, all of its $(q-1)$-tips-and therefore it too-may not exist in $\mathscr{G}_{r}^{p-1}$ for the same reason. If so, remove it from $x^{p}$ as well. If the resulting set $x_{r}^{p}$ has at least one $(p-1)$-tip, it is called a reduced $p$-node (induced by $\mathscr{B}_{r}$ ). Let $\mathscr{N}_{r}^{p}$ be the set of all reduced $p$-nodes. If $\mathscr{N}_{r}^{p}$ is not void, let $\mathscr{G}_{r}$ be the $p$-graph $\left(\mathscr{B}_{r}, \mathscr{N}_{r}^{0}, \ldots, \mathscr{N}_{r}^{p}\right) ;$ if $\mathscr{N}_{r}^{p}$ is void, let $\mathscr{G}_{r}$ be the $(p-1)$-graph $\mathscr{G}_{r}^{p-1}=\left(\mathscr{B}_{r}, \mathscr{N}_{r}^{0}, \ldots, \mathscr{N}_{r}^{p-1}\right)$.

Another situation may arise if $\mathscr{B}_{r}$ is chosen arbitrarily. There may be some integer $q$ with $0 \leq q \leq p-1$ for which the $\mathscr{N}_{r}^{q+1}, \ldots, \mathscr{N}_{r}^{p}$ are all void when the construction of the preceding paragraph is applied recursively with $p$ replaced by $m=1, \ldots, p$. Indeed, if $\mathscr{N}_{r}^{q+1}$ is void, then so too will be $\mathscr{N}_{r}^{m}$ for $m=q+2, \ldots, p$ because representatives of $m$-tips will not exist. In this case, we let $\mathscr{G}_{r}$ be the $q$-graph $\left(\mathscr{B}_{r}, \mathscr{N}_{r}^{0}, \ldots, \mathscr{N}_{r}^{q}\right)$ where $q$ is the largest natural number for which $\mathscr{N}_{r}^{q}$ is not void.

Definition (reduced graph). $\mathscr{G}_{r}$ is called the reduction of $\mathscr{G}^{p}$ with respect to $\mathscr{B}_{r}$ or the reduced graph induced by $\mathscr{B}_{r}$.

Now, consider the $(p-1)$-path

$$
P^{p-1}=\left\{\ldots, x_{m}^{p-1}, P_{m}^{p-2}, x_{m+1}^{p-1}, P_{m+1}^{p-2}, \ldots\right\}
$$

which is an alternating sequence of $(p-1)$-nodes $x_{m}^{p-1},(p-2)$-paths $P_{m}^{p-2}$, and possibly a terminal element-a $q$-node with $q \leq p-1$-to the left and/or to the right. (Here too, this has been explicitly defined for $p-1=1$. In a moment, we shall complete our definition of a higher-rank path by stating the conditions such a path must fulfill. All that need be known right now is that there are entities, called "paths of higher ranks", that are alternating sequences as stated.) $P^{p-1}$ is called nontrivial if it has at least three elements. For larger $p$, each $P_{m}^{p-2}$ can be expanded into another alternating sequence of nodes and paths of still lower rank, and so forth repeatedly.

Definition (to embrace-for a $(p-1)$-path). We say that a $(p-1)$-path embraces itself, and all its elements, as well as the paths of lower ranks, and ultimately 
the branches arising in this repeated expansion of paths. We also say that it embraces all the elements embraced by its nodes and by the nodes arising in this repeated expansion of paths. However, it does not embrace any other entity.

Definition (to meet). If a $p$-node $x^{p}$ embraces a 0 -node $x_{0}^{0}$ belonging to a branch $b$, we say that $b$ meets $x^{p}$ at $x_{0}^{0}$. Now let the integers $n$ and $q$ be no larger than $p-1$. If an $n$-path $P^{n}$ and a $q$-node $x^{q}$ embrace a node in common, then $P^{n}$ and $x^{q}$ are said to meet at that node. Also, with regard to (2) and (4), if a one-ended or endless $(p-1)$-path $P^{p-1}$ contains as a subsequence a representative of a $(p-1)$-tip $t^{p-1}$ in a $p$-node $x^{p}$, then $P^{p-1}$ is said to meet $x^{p}$ with $t^{p-1}$.

For the last situation, it should be noted that, even though $P^{p-1}$ contains a representative of $t^{p-1}$ as a subsequence, $t^{p-1}$ is not an element of $P^{p-1}$, and any node containing $t^{p-1}$ is not embraced by $P^{p-1}$.

Definition (totally disjoint and terminally incident). The $q$-node $x^{q}$ and the $(p-1)$-path $P^{p-1}$ are called totally disjoint if $P^{p-1}$ does not meet $x^{q}$. Also, $x^{q}$ and $P^{p-1}$ are said to be terminally incident if $P^{p-1}$ terminates at a node that embraces or is embraced by $x^{q}$ or if, for $q=p, P^{p-1}$ meets $x^{p}$ with a $(p-1)$-tip in $x^{p}$. Moreover, $x^{q}$ and $P^{p-1}$ are called terminally incident but otherwise totally disjoint if they are terminally incident and $P^{p-1}$ does not meet $x^{q}$ at any other node embraced by $P^{p-1}$ or with any other $(p-1)$-tip. Two $(p-1)$-paths $P_{1}^{p-1}$ and $P_{2}^{p-1}$ are called totally disjoint if the set of all nodes embraced by $P_{1}^{p-1}$ has a void intersection with the set of all nodes embraced by $P_{2}^{p-1}$.

Note that, in order for $P_{1}^{p-1}$ and $P_{2}^{p-1}$ to be totally disjoint, it is not in general sufficient to impose this void-intersection property on just the sets of 0 -nodes embraced by $P_{1}^{p-1}$ and by $P_{2}^{p-1}$. For example, in Figure 1 the two 1-paths $\left\{x_{a}^{0}, P_{a}, x_{1}^{1}, P_{e f}, x_{f}^{0}\right\}$ and $\left\{x_{d}^{0}, P_{d}, x_{2}^{1}\right\}$ embrace nonintersecting 0 node sets. However, they are not totally disjoint because $x_{2}^{1}$ embraces $x_{f}^{0}$.

We now complete our recursive definition of a path of higher rank by explicating the conditions that such a path must satisfy.

Definition ( $p$-path). The alternating sequence

$$
\left\{\ldots, x_{m}^{p}, P_{m}^{p-1}, x_{m+1}^{p}, P_{m+1}^{p-1}, \ldots\right\}
$$

is called a $p$-path if the following three conditions are fulfilled.

(i) Each $P_{m}^{p-1}$ is a nontrivial $(p-1)$-path and each $x_{m}^{p}$ is a $p$-node except possibly when (5) terminates on the left and/or on the right, in which case the terminal element is a $q$-node where $0 \leq q \leq p$.

(ii) Each $P_{m}^{p-1}$ is terminally incident to the two nodes immediately preceding and succeeding it in (5) but is otherwise totally disjoint from those nodes.

(iii) Every two elements in (5) that are not adjacent therein are totally disjoint.

Note that, by designating (5) as a sequence, we understand that the indices $m$ are restricted to the integers; they are not allowed to extend to the transfinite ordinals.

For $p$-paths, the adjectives "nontrivial", "one-ended", and "endless", are defined just as they are for 0-paths. Similarly, a $p$-path is finite if there are only 
a finite number of entries in (5) even though it may be transfinite in the sense that the integers do not suffice to index its embraced branches sequentially in accordance with a tracing of (5). Also, all the terminology used with (4) is carried over to (5).

Definition ( $p$-loop). A $p$-loop is a finite $p$-path except for the following requirement: One of the two terminal elements embraces the other.

Proposition 4.2. Assume that the p-path (5) contains at least one p-node, say $x_{m+1}^{p}$ that is not a terminal element. Then, at least one of the adjacent paths, $P_{m}^{p-1}$ or $P_{m+1}^{p-1}$, meets $x_{m+1}^{p}$ with a $(p-1)$-tip.

Proof. The only way the conclusion can be negated is if both $P_{m}^{p-1}$ and $P_{m+1}^{p-1}$ terminate at the single exceptional element $x_{0}$ in $x_{m+1}^{p}$ in such a way that $P_{m}^{p-1}$ has a terminal element $y_{m}$ and $P_{m+1}^{p-1}$ has a terminal element $y_{m+1}$, each of which embraces or is embraced by $x_{0}$. Three cases arise:

(1) $y_{m}$ and $y_{m+1}$ both embrace $x_{0}$. By Proposition 4.1, either $y_{m}$ embraces $y_{m+1}$ or $y_{m+1}$ embraces $y_{m}$.

(2) $y_{m}$ embraces $x_{0}$ and $x_{0}$ embraces $y_{m+1}$ (or conversely). By definition, $y_{m}$ embraces all the elements embraced by $x_{0}$. Hence, $y_{m}$ embraces $y_{m+1}$. (Conversely, $y_{m+1}$ embraces $y_{m}$.)

(3) $x_{0}$ embraces both $y_{m}$ and $y_{m+1}$. We now invoke the fact that $x_{0}$ contains as an element of itself no more than one exceptional element $w$, and the rank of $w$ is lower than the rank of $x_{0}$. Moreover, $w$ contains no more than one exceptional element $u$, and $u$ is of still lower rank. Continuing in this way, we find that $x_{0}$ and all its embraced exceptional elements form a sequence $\left\{x_{0}, w, u, \ldots\right\}$ whose elements have stricly decreasing ranks. So, $y_{m}$ and $y_{m+1}$ must appear in this sequence. This implies that $y_{m}$ embraces $y_{m+1}$, or conversely.

In all three cases, we obtain a contradiction to the fact that $P_{m}^{p-1}$ and $P_{m+1}^{p-1}$ are totally disjoint.

If (5) terminates on the left (or right) at $x_{a}$, then the $(p-1)$-path $P_{m}^{p-1}$ of lowest (of highest) index $m$ will be called the leftmost (or rightmost) subpath of rank $p-1$ embraced by (5). Similarly, the $(p-2)$-path in that leftmost (rightmost) subpath of lowest (of highest) index, if it exists, will be called the leftmost (or rightmost) subpath of rank p-2 embraced by (5). This terminology is extended to subpaths of still lower rank.

Proposition 4.3. If a p-path $P^{p}$ terminates on the left (right) at a node $x_{a}$ of rank $q$ where $q<p$, then $P^{p}$ embraces leftmost (rightmost) subpaths of every rank $n$, where $n=q-1, \ldots, p-1$.

Proof. Since $P^{p}$ terminates on, say, the left, it contains a leftmost subpath $P^{p-1}$ of rank $p-1$. If $P^{p-1}$ does not contain a leftmost subpath of rank $p-2$, then it can meet $x_{a}$ only with a $(p-1)$-tip. Hence, $x_{a}$ must be of rank $p$ at least. Thus, if $x_{a}$ 's rank is less than $p, P^{p-1}$ must contain a leftmost subpath of rank $p-2$. This argument can be continued inductively to obtain the proposition.

Note. Since $P^{p}$ may terminate at $x_{a}^{q}$ with an $i$-node $y^{i}$, where $i<q$ and $x_{a}^{q}$ embraces $y^{i}$, the conclusion of the last proposition may also hold for some values of $n$ smaller than $q-1$. 
Definition ( $q$-connected). Let the integers $m, n$, and $q$ be no larger than $p$. Let $x_{a}^{n}$ be an $n$-node and $x_{b}^{m}$ be an $m$-node. $x_{a}^{n}$ and $x_{b}^{m}$ are said to be $q$-connected if there exists a finite $q$-path that meets $x_{a}^{n}$ and $x_{b}^{m}$. Two branches are called $q$-connected if their 0 -nodes are $q$-connected. A graph is called $q$-connected if all its branches are $q$-connected.

Proposition 4.4. If two nodes in a p-graph are $q$-connected, then they are $n$ connected for each $n=q+1, \ldots, p$.

Proof. Let

$$
P^{q}=\left\{x_{a}^{l}, P_{0}^{q-1}, x_{1}^{q}, P_{1}^{q-1}, \ldots, P_{i}^{q-1}, x_{b}^{m}\right\}
$$

be a finite $q$-path connecting the two nodes $x_{a}^{l}$ and $x_{b}^{m}$. Thus, both $l$ and $m$ are no larger than $q$. Then, $P^{q+1}=\left\{x_{a}^{l}, P_{q}, x_{b}^{m}\right\}$ is a finite $(q+1)$-path, $P^{q+2}=\left\{x_{a}^{l}, P^{q+1}, x_{b}^{m}\right\}$ is a finite $(q+2)$-path, and so forth.

Definition ( $q$-section). For any $q$ with $0 \leq q \leq p$, a $q$-section of a $p$-graph $\mathscr{G}$ is a reduction of $\mathscr{G}$ induced by a maximal set of branches that are pairwise $q$-connected.

Proposition 4.5. An n-path can pass into or out of a q-section $S^{q}$ (i.e., it embraces a branch in $S^{q}$ and a branch not in $S^{q}$ ) only if $n>q$.

Proof. Suppose this is not so. Then, there will be an $n$-path $P^{n}$ with $n \leq q$ which terminates at both ends at 0 -nodes having incident branches $b_{a}$ and $b_{b}$ lying only in different $q$-sections. Hence, $P^{n}$ is a finite $n$-path. Thus, $b_{a}$ and $b_{b}$ are $n$-connected and, by Proposition 4.4, $q$-connected. By the maximality condition of $q$-sections, $b_{a}$ and $b_{b}$ lie in the same $q$-section, a contradiction.

The last proposition implies that, if $n \leq q$, any $n$-path or $n$-loop is confined to a single $q$-section. On the other hand, the condition $n>q$ is not in general sufficient for the existence of an $n$-path passing through two given $q$-sections because nodes of rank larger than $q$ may not be suitably located in $\mathscr{G}$.

\section{5. $\omega$-GRAPHS}

The next step in generalization occurs when $p$ is replaced by the least transfinite ordinal $\omega$; it requires some modifications in our constructions. We start with a graph that has $p$-nodes for every natural number $p$. Such a graph can be obtained by repeating without end the recursion through which a $p$-graph is constructed. It is specified by the infinite set

$$
\left(\mathscr{B}, \mathscr{N}^{0}, \mathscr{N}^{1}, \ldots\right),
$$

where now the listing of the $\mathscr{N}^{p}$ continues through all the natural numbers $p$. Each $\mathscr{N}^{p}$ is required to be a nonvoid set (and in fact an infinite set, for otherwise $\mathscr{N}^{p+1}$ would be void).

Definition ( $\vec{\omega}$-graph). We call (6) an $\vec{\omega}$-graph and denote it by $\mathscr{G}^{\vec{\omega}}$.

Definition ( $\vec{\omega}$-path). An $\vec{\omega}$-path is a one-ended sequence of the form

$$
P^{\vec{\omega}}=\left\{x_{0}^{q_{0}}, P_{0}^{p_{0}-1}, x_{1}^{p_{1}}, P_{1}^{p_{1}-1}, x_{2}^{p_{2}}, P_{2}^{p_{2}-1}, \ldots\right\}
$$


where $x_{0}^{q_{0}}$ is a $q_{0}$-node, $x_{m}^{p_{m}}$ is a $p_{m}$-node, $P_{m}^{p_{m}-1}$ is a nontrivial $\left(p_{m}-1\right)$ path, $q_{0} \leq p_{0}$, the $p_{m}$ are strictly increasing (i.e., $p_{0}<p_{1}<p_{2}<\cdots$ ), and the members of (7) are pairwise totally disjoint except for adjacent members, which are terminally incident but otherwise totally disjoint.

As a consequence of these conditions, at least one of the two paths $P_{m}^{p_{m}-1}$ and $P_{m+1}^{p_{m+1}-1}$ meets $x_{m+1}^{p_{m+1}}$ with a $\left(p_{m+1}-1\right)$-tip (see Proposition 4.2, whose proof applies just as well to the present situation).

Definition ( $\vec{\omega}$-tip). Two $\vec{\omega}$-paths are called equivalent if they have representatives such as (7) that differ by no more than a finite number of members. Then, an $\vec{\omega}$-tip $t^{\vec{\omega}}$ is an equivalence class of pairwise equivalent $\vec{\omega}$ paths. A representative of $t^{\vec{\omega}}$ is any member of the equivalence class.

Assume now that the $\vec{\omega}$-graph (6) has a nonvoid set $\mathscr{T} \vec{\omega}$ of $\vec{\omega}$-tips and choose a partition $\mathscr{T}^{\vec{\omega}}=\bigcup \mathscr{T}_{\tau}^{\vec{\omega}}$. Also, for each index $\tau$ of the partition, let $\mathscr{N}_{\tau}^{\vec{\omega}}$ be either the void set or a singleton whose element is a $q$-node for some natural number $q$.

Definition ( $\omega$-node). For each index $\tau$, the set

$$
x^{\omega}=\mathscr{T}_{\tau}^{\vec{\omega}} \cup \mathscr{N}_{\tau}^{\vec{\omega}}
$$

is called an $\omega$-node, and, if $\mathscr{N}_{\tau}^{\vec{\omega}}$ is not void, its unique element is called the exceptional element of $x^{\omega}$. In addition, every $\omega$-node is required to satisfy Condition $\Gamma^{\omega}$, which is a restriction on its exceptional element reading exactly as does Condition $\Gamma^{p}$ except that $p$ is replaced by $\omega$.

Condition $\Gamma^{\omega}$. If an $\omega$-node contains a $q$-node $(q<\omega)$ as an exceptional element, then that q-node does not appear as the exceptional element of any other $m$-node, where $q<m \leq \omega$.

Definition ( $\omega$-graph). An $\omega$-graph is an infinite totally ordered set

$$
\mathscr{G}^{\omega}=\left(\mathscr{B}, \mathscr{N}^{0}, \mathscr{N}^{1}, \ldots, \mathscr{N}^{\omega}\right)
$$

having $\omega+2$ entries, where $\mathscr{B}$ is a set of branches and each $\mathscr{N}^{q}$ for $q=$ $0, \ldots, \omega$ is a nonvoid specified set of $q$-nodes.

As with any $p$-graph, when $q$ is a natural number, each $\mathscr{N}^{q}$ can be specified only after the $\mathscr{N}^{m}$, for $m=0, \ldots, q-1$, have been specified, and similarly all the $\mathscr{N}^{q}$ have to be specified before $\mathscr{N}^{\omega}$ can be specified.

A reduced graph $\mathscr{G}_{r}$ of an $\omega$-graph induced by a subset $\mathscr{B}_{r}$ of $\mathscr{B}$ is defined exactly as is a reduced graph of a $p$-graph.

Another way of representing a one-ended $\vec{\omega}$-path is obtained by replacing every index $m$ by $-m$ in (7) and in the conditions imposed upon (7). Furthermore upon appending the result to the left of (7) (and striking out the extra $\left.x_{0}^{q_{0}}\right)$, we obtain an endless $\vec{\omega}$-path $P^{\vec{\omega}}$. All the terminology for $p$-paths extend to one-ended and endless $\vec{\omega}$-paths.

Our definition of an $\omega$-path

$$
\left\{\ldots, x_{m}^{\omega}, P_{m}^{\vec{\omega}}, x_{m+1}^{\omega}, P_{m+1}^{\vec{\omega}}, \ldots\right\}
$$

is the same as that of a $p$-path, given in $\S 4$, except that (5) is replaced by (8), $p$ by $\omega$, and $p-1$ by $\vec{\omega}$ (Note that now each $P_{m}^{\vec{\omega}}$ in (8) must be one-ended 
or endless, not finite.) An $\omega$-loop is a finite $\omega$-path except that one of its two terminal nodes embraces the other one.

With these alterations, Proposition 4.1 through 4.3 hold as before except for some obvious modifications. For example, in Proposition 4.3 the values for $n$ are now $q-1, q, q+1, \ldots$, but not $p-1$ because with $p=\omega$ there is no ordinal $p-1$.

As for connectedness, let $x_{a}^{n}$ and $x_{b}^{q}$ be nodes of ranks $n$ and $q$ respectively, where $0 \leq n \leq \omega$ and $0 \leq q \leq \omega$. These nodes are said to be $\omega$-connected if there is a finite $p$-path, where $p \leq \omega$, that meets $x_{a}^{n}$ and $x_{b}^{q}$. Finally, an $\omega$ section is a reduction of $\mathscr{G}$ induced by a maximal set of branches whose incident 0 -nodes are pairwise $\omega$-connected. Such a section will have more significance in graphs of ranks higher than $\omega$.

\section{GRAPHS OF STILL HIGHER RANKS}

With $\omega$-graphs in hand, we can proceed as in $\S 4$ to obtain $(\omega+p)$-graphs for any natural number $p>0$ by using $(\omega+p-1)$-tips to define $(\omega+p)$ nodes. Then, the method of $\S 5$ provides $(\omega+\vec{\omega})$-tips from which $(\omega 2)$-nodes and $(\omega 2)$-graphs can be obtained. This process can be continued to generate $k$-graphs where $k$ is any countable ordinal that can be reached through these recursive constructions. The procedure of $\S 4(\S 5)$ is used when $k$ is a successor ordinal (respectively, limit ordinal).

For example, an $\omega^{2}$-graph can be constructed as follows: Start with oneended paths of the form (7), where now $p_{m}=\omega n_{m}$ and the $n_{m}$ are natural numbers with $n_{0}<n_{1}<n_{2}<\cdots$. Then define $(\omega \vec{\omega})$-tips $t^{\omega \vec{\omega}}$ as equivalence classes of such paths, pairwise differing on no more than a finite number of elements. This leads to $\omega^{2}$-nodes of the form

$$
x^{\omega^{2}}=\mathscr{T}_{\tau}^{\omega \vec{\omega}} \cup \mathcal{N}_{\tau}^{\omega \vec{\omega}}
$$

from which the $\omega^{2}$-graph $\left(\mathscr{B}, \mathscr{N}^{0}, \ldots, \mathscr{N}^{\omega^{2}}\right)$ can be defined.

Thus, we can have transfinite graphs of rank $k$ for quite a range of finite or transfinite ordinals $k$, and $q$-sections may be defined in these graphs for every $q$ from 0 to $k$.

\section{7. $(k, 0)$-PATHS AND TERMINAL BEHAVIOR AT EXTREMITIES}

Again let $p$ be a natural number larger than 0 . Given the $p$-path (5), we can think of each $P_{m}^{p-1}$ being explicitly written out as a $(p-1)$-path. This will yield an expanded display of (5) involving the $p$-nodes $x_{m}^{p}$ and the possible terminal nodes of $(5)$, as well as the $(p-1)$-nodes, possibly other terminal nodes, and $(p-2)$-paths arising from the expansions of all the $P_{m}^{p-1}$ in (5). (For an example wherein $p=4$, see the second line of Figure 2.) If a $P_{m}^{p-1}$ terminates at an $i$-node $d^{i}(i \leq p-1)$ that is embraced by a $p$-node, the notation $d^{i}$ is deleted from the expanded version of (5). No such deletion is needed if $P_{m}^{p-1}$ meets the $p$-node with a $(p-1)$-tip. By virtue of Proposition 4.2, no more than one such deletion need be made at each $p$-node. On the other hand, if $P_{m}^{p-1}$ is a leftmost (rightmost) subpath, its terminal node on the left (right) is compared in rank with the terminal node on the left (right) in (5). If those ranks are the same, the two terminal elements will be identical, according to Proposition 4.1, 
and just one node notation is retained. If not, we discard the node with the lower rank. In this way, no two nodes appear as adjacent terms in the expansion of (5).

The integers may no longer suffice to index consecutively all the terms of this expanded form of (5). Moreover, its terms, when ordered in accordance with this sequence of sequences, are totally ordered but may not be well-ordered. Well-ordering may be absent, for example, when one of the $P_{m}^{p-1}$ is an endless path. We will refer to this expanded form of $(5)$ as a $(p, p-1)$-path and will denote it by $P^{p, p-1}$.

This process can be repeated, as is indicated in Figure 2. An expansion of all the $(p-2)$-paths in the transfinite $(p, p-1)$-path yields a transfinite $(p, p-2)$-path $P^{p, p-2}$. Continuing in this way, we obtain for each $q<p$ the transfinite $(p, q)$-path $P^{p, q}$ and finally a transfinite $(p, 0)$-path $P^{p, 0}$, which is totally ordered but not necessarily well-ordered. The elements of $P^{p, 0}$ will be branches interspersed with nodes of various ranks. Two adjacent branches will be separated by a 0 -node to which they are incident. The higher-order nodes will separate various finite or infinite totally ordered sets.

The $(p, 0)$-loops are defined from the $p$-loops in just the same way and called transfinite loops if they have more than a finite number of branches. Note that, for each $p$-path or $p$-loop, there is a corresponding, uniquely defined $(p, 0)$ path or $(p, 0)$-loop.

An $\omega^{4}$-path: $x_{0}^{2}, P_{0}^{3}, x_{1}^{4}, P_{1}^{3}, x_{2}^{4}, P_{2}^{3}, \cdots$

Its $\omega^{4,3}$-path: $x_{0}^{2}, P_{0}^{2}, x_{1}^{3}, P_{1}^{2}, x_{2}^{3}, \cdots, x_{1}^{4}, d_{Q}^{3}, Q_{0}^{2}, d_{1}^{3}, Q_{1}^{2}, \cdots$

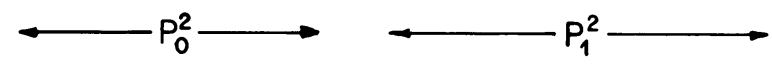

Its $\omega^{4,2}$-path: $x_{0}^{2}, P_{0}^{1}, x_{1}^{2}, P_{1}^{1}, x_{2}^{2}, \cdots, x_{1}^{3}, \cdots, R_{-1}^{1}, e_{0}^{2}, R_{0}^{1}, e_{1}^{2}, \cdots$

Its $\omega^{4,1}$-path: $\quad \stackrel{\longrightarrow}{x_{0}^{2}, \cdots, P_{-1}^{0}, x_{0}^{1}, P_{0}^{0}, x_{1}^{1}, \cdots, x_{1}^{2}, \cdots, S_{-1}^{0}, f_{0}^{1}, S_{0}^{0}, f_{1}^{1}, \cdots}$

Its $\omega^{4,0}$-path: $x_{0}^{2}, \cdots \cdots \cdots, P_{-1}^{0} \rightarrow \stackrel{x_{0}^{1}, \cdots, x_{-1}^{0}, b_{-1}, x_{0}^{0}, b_{0}, \cdots, x_{1}^{1}, \cdots \cdots}{-P_{1}^{0}-}$

FIGURE 2. Illustration of the possible terminal behaviors of a 4-path and its corresponding $(4, q)$-paths $(q=$ $0, \ldots, 3)$. The 4-path is assumed to terminate on the left at a 2-node $x_{0}^{2}$. The $d$ 's, $e$ 's, $f$ 's, and $x$ 's denote nodes, and the $P$ 's, $Q$ 's, $R$ 's, and $S$ 's denote paths of the indicated ranks. The $b$ 's are branches. The terminal element $d_{0}^{i} \quad(i \leq 3)$ on the left-hand side of $P_{1}^{3}$ is deleted in the expansion of $P_{1}^{3}$ in the second line because it is embraced by $x_{1}^{4}$. 
Assume $P^{p}$ is a $p$-path that terminates on the left at a $q$-node $x_{0}^{q}$ where $q<p$. Then, by Proposition 4.3, $P^{p}$ embraces leftmost subpaths $P_{0}^{n}$ of every rank $n$, where $n$ varies from $p-1$ down to $q-1$ and perhaps lower. Let $m$ be the smallest $n$ for which $P^{p}$ embraces a leftmost node of rank $n-1$. (This is illustrated in Figure 2 for $p=4, q=2$, and $m=2$.) In short, there may be a critical value of $n$, namely, $m$ such that $P^{p}$ embraces a leftmost subpath $P_{0}^{n-1}$ of rank $n-1$ for every $n=m, \ldots, p$, but not for $n<m$. This means that $P_{0}^{m}, P_{0}^{m+1}, \ldots, P_{0}^{p}$ all terminate at $x_{0}^{q}$ with a node, that $P_{0}^{m-1}$ meets $x_{0}^{q}$ with an $(m-1)$-tip, and that for $n=0, \ldots, m-2$ there is no embraced $n$-path that terminates at or meets $x_{0}^{q}$.

The same kind of pattern will exist at all the $p$-nodes of $P^{p}$ at which $(p-1)$ subpaths terminate.

Similarly, any $\omega$-path or $\omega$-loop can be expanded into nested sequences of paths and nodes of lower ranks because each $P_{m}^{\vec{\omega}}$ in (8) has the one-ended form (7) or the endless version of (7). This ultimately yields a uniquely defined, totally ordered (but not in general well-ordered) set of branches interspersed with nodes whose ranks vary from 0 to $\omega$; that result will be called an $(\omega, 0)$ path or an $(\omega, 0)$-loop.

These ideas extend directly to $k$-paths, where $k$ is any countable ordinal obtained as indicated in $\S 6$. We obtain thereby $(k, 0)$-paths and $(k, 0)$-loops.

\section{PART II}

\section{TRANSFINITE ELECTRICAL NETWORKS}

Infinite electrical networks have appeared intermittently in both the mathematical and electrical engineering literature for most of this century, but the earlier works were restricted to networks having graphs with regular repetitive patterns, such as ladders and grids. It has been only during the past two decades that networks with arbitrary graphs have been the subject of an ongoing research activity. The seminal work in this area was by Flanders [7] and appeared in 1971. It established an existence and uniqueness theorem for the voltage-current regime in a locally finite, linear, resistive network having only a finite number of sources and open circuits everywhere at infinity. This was followed by a series of papers that generalized the theory in various ways; see [1-4, 19-25], and the references therein. Actually, infinite electrical networks arise in quite a different context as well, namely, in the theory of random walks on infinite graphs $[5,6$, 12, 14, 16-18]. All the infinite electrical networks considered up to now have been finitely connected, that is, between every two nodes there exists a finite path. Nonetheless, infinite networks having some pairs of nodes connected by infinite paths but not by finite ones is an idea worth pursuing.

This paper was inspired by the following question. What kind of connections can be made between the "infinite extremities" of an infinite network? That short circuits as well as pure voltage or current sources can be so connected was established in [21 and 24], but resistances between extremities remained an open problem, which this paper now resolves. Moreover, if resistances can be connected out at infinity, so too can other infinite networks, and we are thereby led naturally to a transfinitely connected infinite network, that is, to a theory of electrical networks based upon $k$-graphs rather than on conventional graphs.

The idea of transfinite electrical networks in the special cases of ladders and 
grids occurs in [26 and 27], but those works use the regular structures of their graphs in essential ways. The arbitrariness of the graphs in this paper requires a much different analysis.

A primary result of this work is an existence and uniqueness theorem, established in $\S 10$, for the voltage-current regime in a transfinite electrical network. The fundamental principle upon which it is based is Tellegen's equation. Kirchhoff's laws follow as a consequence ( $\$ 11)$, but those laws need not hold everywhere in the network. The current law will hold at every "restraining node" and the voltage law will hold around every "perceptible loop." A node is "restraining" if the sum of its incident conductances is finite, and a loop is "perceptible" if the sum of its resistances is finite.

The proof of the existence and uniqueness theorem is roughly analogous to a mesh analysis of a finite network, for the solution space is constructed out of finite and transfinite loop currents. The question naturally arises as to whether a dual theory (dual in the sense of circuit theory) can also be devised. This is accomplished in $\S 12$, where now the fundamental quantities, from which another solution space is built, are sets of branch voltages. This leads to another existence and uniqueness theorem, but it is shown in $\S 13$ that the two theories are equivalent in that they yield the same voltage-current regime.

Another objective of this work is to examine the voltages at the extremities of a transfinite network, that is, at its 0-tips for various sections. Section 14 establishes that tips, which are "not disconnectable" in a certain sense, cannot have different voltages; this implies that Halin's finitely chainlike structure cannot have more than a finite number of different tip voltages. Furthermore, it is shown in $\S 15$ that various 0 -tip voltages may be forced to be identical because of a particular distribution of resistance values, even when the tips are disconnectable.

In the last category of results, we have a generalization of Thomson's least power principle [9, p. 322] for transfinite networks; it is given in $\S 16$. Also, the concavity of driving-point resistance or conductance functions is established in $\S 17$; the special case for finite networks was given in [11] and the special case for infinite (but not transfinite) networks was given in [4]. All these concavity theorems are generalizations of the result originated by Shannon and Hagelbarger [15].

Finally, this paper provides a basis for a theory of transfinite random walks, wherein the walker wanders along transfinite paths, possibly "passing through infinity" many times [28, 29].

Example. Consider the infinite ladder network of Figure 3 having the indicated resistance values. The upper nodes are indexed consecutively from left to right by the natural numbers. In analogy to a finite ladder network, we might suppose that output terminals exist at the end of this infinite ladder network, namely, at the 1 -nodes indicated by the small circles in Figure 3. If so, a load resistance $R_{L}$ might be connected thereto. Let us suppose still further that the monotonicity principle for resistance functions continues to hold for this ladder network. Consequently, the driving-point resistance $R_{D}$ should be less than the value it becomes when all the shunting resistance values are replaced by $\infty$. So, when $R_{L}=0$, this results in an infinite series circuit and $R_{D}<.111 \cdots=1 / 9$. On the other hand, $R_{D}$ should be larger than the value it becomes when all the series 


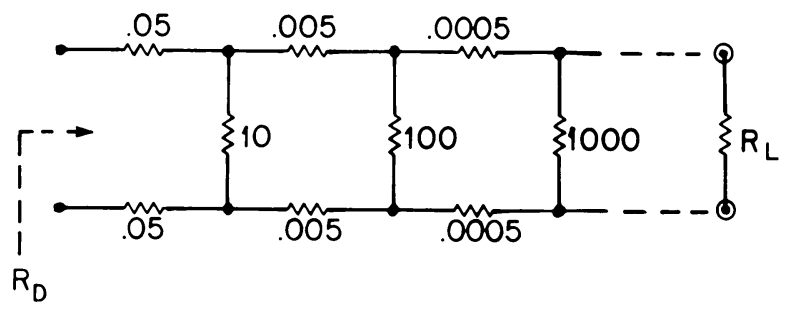

FiguRE 3. An infinite ladder network whose drivingpoint resistance $R_{D}$ as seen from the input terminals on the left depends on the load resistance $R_{L}$ connected on the right to the extremities of the network represented by two 1-nodes at infinity shown by the small circles. The numbers are resistance values in ohms, which continue in the indicated pattern infinitely to the right.

resistances are replaced by 0 . Now, for $R_{L}=\infty$, we obtain an infinite parallel circuit and $R_{D}>1 / .111 \cdots=9$. We could then conclude that $R_{D}$ changes when $R_{L}$ changes, and so we might infer that, in order for a voltage-current regime to be determined when a source is impressed at the input to the ladder, we must specify what the connection at infinity is-at least for this particular network. In short, infinity is "perceptible" to an observer at the input. This heuristic argument for ladder networks has a completely rigorous justification [26].

The point here is that we now have a network with a connection "at infinity", in particular, a resistance $R_{L}$ whose nodes are embraced by two 1-nodes. Moreover, $R_{L}$ may be replaced by the input to another infinite ladder network to get a network that extends "beyond infinity." In short, an electrical network theory based upon $k$-graphs is needed.

\section{9. $k$-NETWORKS}

Now that we have constructed transfinite graphs, we shall assign an analytical structure to every branch to obtain thereby transfinite electrical networks. First of all, note that, since $\mathscr{B}$ is a countable set, the natural numbers suffice to index all the branches of a given $k$-graph $\mathscr{G}$ in some fashion. (It is when we try to index the branches of a $(k, 0)$-path in the order of a tracing along that path that the natural numbers or even a well-ordered indexing system may not suffice.) Henceforth, we assume that every branch has a natural number $j$ as an index, where $j=0,1,2, \ldots$. Furthermore, we assume that every branch has an orientation, with respect to which the polarities of voltages and currents will be measured.

The $j$ th branch's analytical structure is given by Thevenin's circuit, shown in Figure 4, where a pure voltage source of value $e_{j}$ volts and a resistance of value $r_{j}$ ohms are connected in series. $e_{j}$ is a real number, possibly zero, and $r_{j}$ is a real positive number. $g_{j}$ will always denote the branch conductance $1 / r_{j}$. Ohm's law and Kirchhoff's laws dictate that

$$
v_{j}=r_{j} i_{j}-e_{j},
$$

where $v_{j}$ is the value of the branch voltage and $i_{j}$ is the value of the branch 


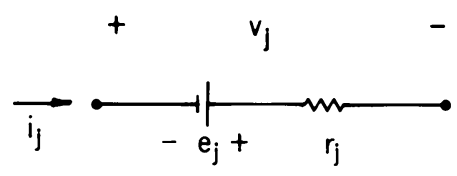

FIgURE 4. Thevenin's circuit for the $j$ th branch. The branch's orientation is taken to be in the direction of $i_{j}$ and in accordance with the polarities of $v_{j}$ as a voltage drop and of $e_{j}$ as a voltage rise.

current, both being real quantities. (To simplify notation, we will use $r_{j}$ to designate the resistor as well as its resistance value, and similarly for $e_{j}, v_{j}$, and $i_{j}$. ) In accordance with this analytical structure, we will only examine purely resistive networks having no dependent sources.

Definition ( $k$-network). An electrical network of rank $k$ or simply a $k$-network is taken to mean a $k$-graph every branch of which has the analytical representation shown in Figure 4 with its parameters satisfying (9).

Henceforth, the symbol $\sum$ will denote a summation $\sum_{j=0}^{\infty}$ over all the branch indices $j$ unless something else is explicitly indicated. We shall impose

Condition $\mathbf{E}$. The branch voltage sources $e_{j}$ all taken together satisfy the condition of finite total isolated power, namely, $\sum e_{j}^{2} g_{j}<\infty$.

As we shall see below, the total power absorbed in all the resistors, which will equal the total power delivered by all the sources, is no larger than $\sum e_{j}^{2} g_{j}$. Thus, Condition $\mathrm{E}$ implies that the $k$-network will be in a finite-power regime.

\section{THE UNIQUe VOLTAGE-CURRENT REGIME}

Boldface notation will denote one-way infinite vectors whose elements are indexed by the natural numbers; thus, $\mathbf{i}=\left(i_{0}, i_{1}, i_{2}, \ldots\right)$ is the vector of all branch currents, $\mathbf{v}=\left(v_{0}, v_{1}, v_{2}, \ldots\right)$ is the vector of all branch voltages, and $\mathbf{e}=\left(e_{0}, e_{1}, e_{2}, \ldots\right)$ is the vector of all branch voltage-source values. $R$ will denote the resistance operator that assigns to every branch-current vector $\mathbf{i}$ the vector $R \mathbf{i}=\left(r_{0} i_{0}, r_{1} i_{1}, r_{2} i_{2}, \ldots\right)$ consisting of the voltages across the branch resistances (i.e., the voltage drops $r_{j} i_{j}$ measured in the direction of the branches' orientations). The support of any vector of branch quantities is the set of all branches for which those quantities are nonzero.

$\mathscr{I}$ denotes the space of all branch-current vectors $\mathbf{i}$ for which $\sum i_{j}^{2} r_{j}<\infty$, that is, for which the total power dissipated in all the resistors is finite. The linear operations are defined componentwise on the vectors i. Moreover, we assign the inner product $(\mathbf{i}, \mathbf{s})$ to two elements $\mathbf{i}, \mathbf{s} \in \mathcal{F}$, where $(\mathbf{i}, \mathbf{s})=$ $\sum r_{j} i_{j} s_{j} ;\|\cdot\|$ denotes the corresponding norm. A standard argument $[10, \mathrm{p}$. 21] shows that $\mathscr{I}$ is complete under this norm and is therefore a Hilbert space and that convergence in $\mathscr{I}$ implies componentwise convergence.

The next step is to assign currents to various $q$-loops, where $q \leq k$, or more precisely to the branches of the corresponding $(q, 0)$-loops. We now assign an orientation to every $q$-loop and thereby to the corresponding $(q, 0)$-loop; it is one of the two possible ways of tracing around the loop. 
Definition ( $q$-loop current). A $q$-loop current or just loop current is an assignment of branch currents such that the currents $i_{j}$ in all branches are zero except for the branches in the corresponding $(q, 0)$-loop; in those latter branches the currents are $i_{j}= \pm i$, where $i$ is a real constant and the plus (minus) sign is used if the $j$ th branch's orientation agrees (respectively, disagrees) with the orientation of the loop.

Kirchhoff's current law asserts that, given a 0-node $x_{0}^{0}$,

$$
\sum_{j \in N} \pm i_{j}=0
$$

where $N$ is the branch-index set for all the branches incident to $x_{0}^{0}, i_{j}$ is the branch current in branch $b_{j}, j \in N$, and the plus (minus) sign is used if $b_{j}$ is oriented toward (away from) the node. If branch $b_{j}$ is a selfloop (i.e., if it is incident to just one node), $j$ appears twice among the indices of the summation, and $+i_{j}$ and $-i_{j}$ both appear in the summation; this will be significant when we consider the absolute convergence of Kirchhoff's current law. A loop current will satisfy Kirchhoff's current law at every 0 -node except possibly when the node is embraced by a $q$-node $x^{q}$ where $q>0$. In the latter case, Kirchhoff's current law will still be satisfied if the corresponding $(q, 0)$-loop passes from one branch $b_{1}$ incident to the 0 -node to another branch incident to the 0 -node but will not be satisfied if the loop passes from $b_{1}$ to a tip embraced by $x^{q}$.

Definition (ordinary 0-node). A 0-node (finite or infinite) will be called ordinary if it is not embraced by any $q$-node where $q>0$.

Definition (perceptible $q$-loop). A $q$-loop $L$ will be called perceptible if $\sum_{j \in \Lambda} r_{j}$ $<\infty$ where $\Lambda$ is the index set for all the branches embraced by $L$.

It follows immediately that a loop current will be a member of $\mathscr{I}$ if and only if its loop is perceptible.

Definition ( $q$-basic current). Let $q$ be any ordinal no larger than $k$. A $q$-basic current is current vector $\mathbf{i}$ of the form $\mathbf{i}=\sum \mathbf{i}_{n}$ satisfying the following four conditions:

(i) The set of summands $\mathbf{i}_{n}$ is finite or denumerable.

(ii) Each $i_{n}$ is a $q$-loop current such that the $q$-loop is not an $l$-loop for any $l<q$.

(iii) The support of $\mathbf{i}$ has a finite $q$-diameter, that is, there exists a natural number $d$ such that every two branches in that support are connected by a $q$-path having no more than $d$-nodes.

(iv) Every ordinary 0-node is embraced by no more than a finite number of the $q$-loops corresponding to the $\mathbf{i}_{n}$. (In other words, only a finite number of the $\mathbf{i}_{n}$ "flow through" any given ordinary 0-node.)

It is possible for a $q$-basic current to be a member of $\mathscr{I}$ without any of its summands $\mathbf{i}_{n}$ being in $\mathscr{I} ; \S \mathrm{XI}$ of [24] gives an example of this for the case where $q=1$.

For a given $k$-network, $\mathscr{K}^{0}$ will denote the span of all $q$-basic currents, where $0 \leq q \leq k$, that are members of $\mathscr{I}$. Thus, $\mathscr{K}^{0} \subset \mathscr{I}$. $\mathscr{K}$ will denote the closure of $\mathscr{K}^{0}$ in $\mathscr{I}$, and so $\mathscr{K} \subset \mathscr{I}$ as well. In fact, $\mathscr{K}$ is a Hilbert 
space by itself when it is equipped with the inner product of $\mathscr{I}$. Moreover, convergence in $\mathscr{K}$ implies componentwise (i.e., branchwise) convergence.

Example. Actually, enlarged $q$-nodes may effectively be introduced when taking the closure of $\mathscr{K}^{0}$. For example, refer to the 1-graph of Figure 5. Assume that the branch resistance values decay so rapidly as one proceeds to the right that $\sum r_{j}<\infty$. Figure 5(a) shows a 1-loop current consisting of a flow along the branches $a_{m}$, through a 1-node $c^{1}=\left\{x^{0}, t_{a}^{0}\right\}$, and back through a single return branch; $x^{0}$ is a 0-node, to which the return branch is incident, and $t_{a}^{0}$ is the 0 -tip having as a representative the 0-path of $a_{m}$ branches. Since this 1-loop is perceptible, its loop current is a member of $\mathscr{K}^{0}$. Also, all the 0-loop currents of Figure 5(b) are members of $\mathscr{K}^{0}$ too. Furthermore, we assume that the 0-tip $t_{b}^{0}$ corresponding to the $b_{m}$ branches comprises a singleton 1-node. However, if all the indicated loop currents of Figures 5(a) and 5(b) have 1-ampere values,

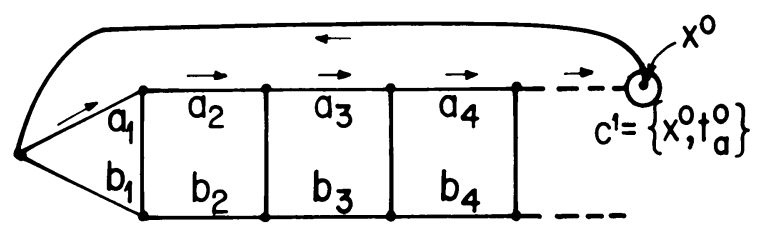

(a)

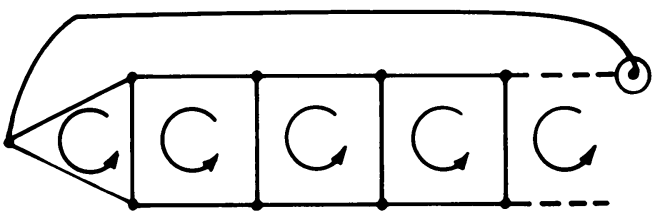

(b)

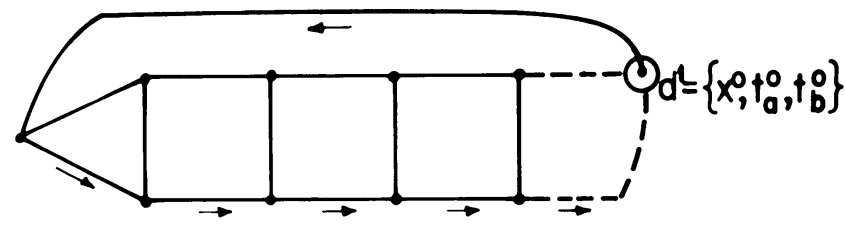

(c)

Figure 5. Assume that the sum of all resistance values is finite, that $c^{1}$ is a 1 -node, but that $d^{1}$ is not specified as a 1 -node. The 1-ampere loop currents shown in parts (a) and (b) are members of $\mathscr{K}^{0}$. Consequently, $\mathscr{K}$ contains the loop current shown in part (c), which means that a new 1-node $d^{1}$, an enlargement of $c^{1}$, has been introduced in effect just by taking the closure of $\mathscr{K}^{0}$. 
their superposition will be the 1-ampere 1-loop current shown in Figure 5(c) and will be a member of $\mathscr{K}$ too. Thus, an enlarged 1-node $d^{1}=\left\{x^{0}, t_{a}^{0}, t_{b}^{0}\right\}$ has effectively been introduced by taking the closure of $\mathscr{K}^{0}$, even though $d^{1}$ was not declared to be a 1-node for this 1-graph. In fact, by appropriately altering the directions of the loop currents of Figure 5(b), we see that every 0-tip is in effect included in an enlarged 1-node.

We now turn to the voltage sources. Any branch voltage-source vector $\mathbf{e}$ defines a mapping (i.e., a functional) from $\mathscr{K}$ into the real line $R^{1}$ according to

$$
\langle\mathbf{e}, \mathbf{i}\rangle=\sum e_{j} i_{j}, \quad \mathbf{i} \in \mathscr{K},
$$

whenever $\sum e_{j} i_{j}$ converges.

Lemma 10.1. If e satisfies Condition $\mathrm{E}$, then $\mathrm{e}$ defines a continuous linear mapping of $\mathscr{K}$ into $R^{1}$ according to (11).

Proof. We first show that $\sum e_{j} i_{j}$ converges absolutely. By Schwarz's inequality,

$$
\sum\left|e_{j} i_{j}\right|=\sum\left|g_{j}^{1 / 2} e_{j} r_{j}^{1 / 2} i_{j}\right| \leq\left[\sum g_{j} e_{j}^{2} \sum r_{j} i_{j}^{2}\right]^{1 / 2} .
$$

The right-hand side is finite by virtue of Condition $\mathrm{E}$ and the fact that $\mathbf{i} \in \mathscr{K}$.

Since absolutely convergent series can be rearranged, the functional defined by (11) is linear. Moreover, it is continuous because, according to (12) and the norm of $\mathscr{K}$,

$$
|\langle\mathbf{e}, \mathbf{i}\rangle| \leq \sum\left|e_{j} i_{j}\right| \leq\left[\sum g_{j} e_{j}^{2}\right]^{1 / 2}\|\mathbf{i}\| .
$$

Here is a fundamental existence and uniqueness theorem for the voltagecurrent regime of a $k$-network.

Theorem 10.2. Given a $k$-network with a branch voltage-source vector e that satisfies Condition $\mathrm{E}$, there exists a unique $\mathbf{i} \in \mathscr{K}$ such that

$$
\langle\mathbf{e}-R \mathbf{i}, \mathbf{s}\rangle=0
$$

for every $s \in \mathscr{K}$. This equation implies the uniqueness of $\mathbf{i}$ in $\mathscr{K}$ even when $\mathbf{s}$ is restricted to $\mathscr{K}^{0}$.

Proof. Since e defines a continuous linear functional on $\mathscr{K}$ according to Lemma 10.1, we can invoke the Riesz representation theorem to conclude that there is a unique $\mathbf{i} \in \mathscr{K}$ such that $\langle\mathbf{e}, \mathbf{s}\rangle=(\mathbf{s}, \mathbf{i})$. On the other hand, $(\mathbf{s}, \mathbf{i})=\sum r_{j} s_{j} i_{j}=\langle R \mathbf{i}, \mathbf{s}\rangle$. Thus, (13) holds for that unique $\mathbf{i}$. Moreover, $\mathbf{i}$ is uniquely determined as a member of $\mathscr{K}$ by the values of $(\mathbf{s}, \mathbf{i})$ for all $\mathbf{s} \in \mathscr{K}$, and in fact for just all the $\mathbf{s} \in \mathscr{K}^{0}$ since $\mathscr{K}^{0}$ is dense in $\mathscr{K}$.

Equation (13) is known in the electrical engineering literature as Tellegen's equation. It, rather than Kirchhoff's laws, is the governing equation that determines the voltage-current regime for our $k$-network. Actually, the uniqueness of that regime arises from the conjunction of the finite-total-isolated-power condition (Condition $\mathrm{E}$ ), the restriction of the allowable branch-current vectors to $\mathscr{K}$, and Tellegen's equation (13). Nonetheless, as we shall see in the next section, Kirchhoff's laws do hold in certain circumstances, even though they have been relegated to a secondary role in this theory. Also, Ohm's law has been imposed upon every $r_{j}$ by virtue of the term $R \mathbf{i}$ in (13). 
Corollary 10.3. Under the hypothesis of Theorem 10.2, the total power $\sum i_{j}^{2} r_{j}$ dissipated in all the resistors equals the total power $\sum e_{j} i_{j}$ supplied by all the voltage sources and is no larger than the finite total isolated power $\sum e_{j}^{2} g_{j}$ available from all the voltage sources.

Proof. Set $\mathbf{s}=\mathbf{i}$ in (13) to get

$$
\sum i_{j}^{2} r_{j}=\langle R \mathbf{i}, \mathbf{i}\rangle=\langle\mathbf{e}, \mathbf{i}\rangle=\sum e_{j} i_{j} .
$$

Upon combining this with (12), we obtain $\sum i_{j}^{2} r_{j} \leq \sum e_{j}^{2} g_{j}$.

\section{KIRCHHOFF'S LAWS}

Definition (restraining 0-node). A 0-node $x_{0}^{0}$ is called restraining if the sum of the conductances of all the branches incident to $x_{0}^{0}$ is finite, that is, if $\sum_{j \in N} g_{j}<\infty$ where $N$ is the index set for all the branches incident to $x_{0}^{0}$. In the case of a self-loop, $g_{j}$ appears twice in the summation. ing.

A finite node is restraining, but an infinite node may or may not be restrain-

Theorem 11.1. If $x_{0}^{0}$ is an ordinary restraining 0-node, then, under the voltagecurrent regime dictated by Theorem 10.2, Kirchhoff's current law (10) is satisfied at $x_{0}^{0}$; moreover, the series on the left-hand side of (10) converges absolutely when $x_{0}^{0}$ is an infinite node.

Proof. Let $\sum_{N}$ denote $\sum_{j \in N}$, and let $\mathbf{i} \in \mathscr{K}$. Then,

$$
\sum_{N}\left|i_{j}\right|=\sum_{N} r_{j}^{1 / 2}\left|i_{j}\right| g_{j}^{1 / 2} \leq\left[\sum_{N} r_{j} i_{j}^{2} \sum_{N} g_{j}\right]^{1 / 2} \leq\|\mathbf{i}\|\left[\sum_{N} g_{j}\right]^{1 / 2} .
$$

Since $x_{0}^{0}$ is restraining, the right-hand side is finite, which establishes the asserted absolute convergence.

Next, as was noted above, every loop current and therefore every basic current satisfies (10) at $x_{0}^{0}$. Consequently, so too does every member of $\mathscr{K}^{0}$ because each such member is a (finite) linear combination of loop currents. Since $\mathscr{K}^{0}$ is dense in $\mathscr{K}$, we can choose a sequence $\left\{\mathbf{i}_{m}\right\}_{m=0}^{\infty}$ in $\mathscr{K}^{0}$ which converges in $\mathscr{K}$ to the unique $\mathbf{i} \in \mathscr{K}$ specified in Theorem 10.2. Thus, with $i_{m j}$ (or $i_{j}$ ) denoting the $j$ th component of $\mathbf{i}_{m}$ (of $\mathbf{i}$ ), we may write $\sum_{j \in N} \pm i_{m j}=0$ and

$$
\begin{aligned}
\left|\sum_{j \in N} \pm i_{j}\right| & =\left|\sum_{N} \pm i_{j}-\sum_{N} \pm i_{m j}\right| \leq \sum_{N}\left|i_{j}-i_{m j}\right|=\sum_{N} r_{j}^{1 / 2}\left|i_{j}-i_{m j}\right| g_{j}^{1 / 2} \\
& \leq\left[\sum_{N} r_{j}\left(i_{j}-i_{m j}\right)^{2} \sum_{N} g_{j}\right]^{1 / 2} \leq\left\|\mathbf{i}-\mathbf{i}_{m}\right\|\left(\sum_{N} g_{j}\right)^{1 / 2} \rightarrow 0
\end{aligned}
$$

as $m \rightarrow \infty$. Thus, $\mathbf{i}$ satisfies Kirchhoff's current law at $x_{0}^{0}$.

Consider now Kirchhoff's voltage law. This asserts that

$$
\sum_{j \in \Lambda} \pm v_{j}=0
$$


where $\Lambda$ is the branch-index set for all the branches embraced by a given oriented $q$-loop $L, v_{j}$ is the branch voltage in branch $b_{j}, j \in \Lambda$, and the plus (minus) sign is used if the orientation of $b_{j}$ agrees (disagrees) with the orientation of loop $L$. (Note that $L$ bequeaths its orientation to the corresponding $(q, 0)$-loop in an obvious way and therefore the two orientations can be compared.)

Definition (perceptible $q$-loop). A $q$-loop $L$ is called perceptible if $\sum_{j \in \Lambda} r_{j}<$ $\infty$.

Theorem 11.2. If $L$ is a perceptible q-loop, then, under the voltage-current regime dictated by Theorem 10.2, Kirchhoff's voltage law (14) holds around $L$, and the series on the left-hand side of (14) converges absolutely.

Proof. Let $\mathbf{s}$ be the $q$-loop current corresponding to a unit current flow around $L$. Since $v_{j}=r_{j} i_{j}-e_{j}$ for each branch, the substitution of $\mathbf{s}$ into (13) yields (14).

Let us now show that the left-hand side of (14) converges absolutely. As before, $\sum_{\Lambda}$ will denote $\sum_{j \in \Lambda}$. We may write

$$
\sum_{\Lambda}\left|e_{j}\right|=\sum_{\Lambda} r_{j}^{1 / 2}\left|e_{j}\right| g_{j}^{1 / 2} \leq\left[\sum_{\Lambda} r_{j} \sum_{\Lambda} e_{j}^{2} g_{j}\right]^{1 / 2} \text {. }
$$

By Condition $\mathrm{E}$ of $\S 9$ and the perceptibility of $L$, the right-hand side is finite. Similarly,

$$
\sum_{\Lambda}\left|r_{j} i_{j}\right|=\sum_{\Lambda} r_{j}^{1 / 2}\left|i_{j}\right| r_{j}^{1 / 2} \leq\left[\sum_{\Lambda} r_{j} i_{j}^{2} \sum_{\Lambda} r_{j}\right]^{1 / 2} .
$$

Since $\mathbf{i} \in \mathscr{K}$ and $L$ is perceptible, the last right-hand side is finite too. Since $v_{j}=r_{j} i_{j}-e_{j}$, we are done.

\section{A DUAL ANALYSIS}

For finite networks mesh and nodal analysis are "dual" in the circuit-theory sense, that is, currents play the fundamental role in mesh analysis whereas voltages do the same in nodal analysis. The question naturally arises as to whether Theorem 10.2 has a dual in a similar sense. In response we present in this section an existence-and-uniqueness theorem based upon voltage vectors.

First of all, we transform every branch from the Thevenin form shown in Figure 4 to the equivalent Norton form shown in Figure 6, where $g_{j}=1 / r_{j}$ and $h_{j}=-g_{j} e_{j}$. This amounts to rewriting (9) as $i_{j}=g_{j} v_{j}-h_{j} . \mathbf{h}=$ $\left(h_{0}, h_{1}, h_{2}, \ldots\right)$ denotes the branch current-source vector. We shall assume that $h$ satisfies

Condition H. $\sum h_{j}^{2} r_{j}<\infty$.

This is equivalent to Condition $\mathrm{E}$ because $\sum h_{j}^{2} r_{j}=\sum\left(h_{j} r_{j}\right)^{2} g_{j}=\sum e_{j}^{2} g_{j}$. Given $\mathbf{h}$, we search for a solution for the voltage vector $\mathbf{v}$ satisfying another generalized form of Tellegen's equation (see (15) below). That vector is also required to be of finite power. This is ensured by requiring the voltage vector to be a member of the space $\mathscr{V}$, which is defined as the space of all $\mathbf{v}=$ 


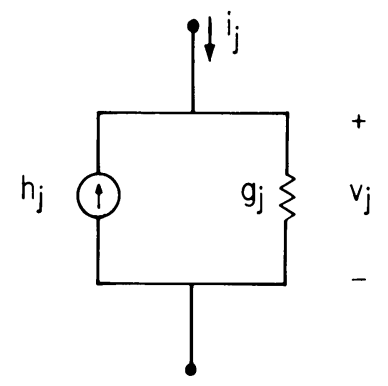

FIGURE 6. The Norton's equivalent form of the $j$ th branch. It is understood that $h_{j}, i_{j}$, and $v_{j}$ have the relative polarities shown and that the branch is oriented in the direction of $i_{j}$.

$\left(v_{0}, v_{1}, v_{2}, \ldots\right)$ such that $\sum v_{j}^{2} g_{j}<\infty$ and $\langle\mathbf{v}, \mathbf{s}\rangle=\sum v_{j} s_{j}=0$ for all $\mathbf{s} \epsilon$ $\mathscr{K}^{0}$. This last condition insures in addition that Kirchhoff's voltage law is satisfied around every perceptible $q$-loop $(0 \leq q \leq k)$ but is actually a stronger condition. The linear operations are defined on $\mathscr{V}$ branchwise. We assign to $\mathscr{V}$ the inner product $(\mathbf{v}, \mathbf{w})=\sum g_{j} v_{j} w_{j}$ for all $\mathbf{v}, \mathbf{w} \in \mathscr{V} .\|\cdot\|$ denotes the corresponding norm. Standard arguments $[10$, p. 21] show that $\mathscr{V}$ is complete and therefore a Hilbert space. The conductance operator $G$ assigns to each $\mathbf{v} \in \mathscr{V}$ the current vector $G \mathbf{v}=\left(g_{0} v_{0}, g_{1} v_{1}, g_{2} v_{2}, \ldots\right)$.

With these definitions in hand, we may repeat the proofs of Lemma 10.1 and Theorem 10.2, interchanging voltages and currents as well as resistances and conductances, to obtain a result dual to Theorem 10.2.

Theorem 12.1. Let there be given a k-network with branches in Norton's form and let $\mathbf{h}$ satisfy Condition $\mathbf{H}$. Then, there exists a unique $\mathbf{v} \in \mathscr{V}$ such that

$$
\langle\mathbf{w}, \mathbf{h}-G \mathbf{v}\rangle=0
$$

for every $\mathbf{w} \in \mathscr{V}$.

\section{The EQUiVAlenCE OF THE DUAL APPROACHES}

We now prove in two steps that Theorems 10.2 and 12.1 are equivalent in the sense that they yield the same voltage-current regime.

Theorem 13.1. Let $\mathbf{i}$ be dictated by Theorem 10.2 and set $\mathbf{v}=R \mathbf{i}-\mathbf{e}$. Then, $\mathbf{v}$ is the unique member of $\mathscr{V}$ specified by Theorem 12.1 .

Proof. The first fact to note is that e satisfies Condition $\mathrm{E}$ if and only if $\mathbf{h}$ satisfies Condition $\mathrm{H}$; this was pointed out just after Condition $\mathrm{H}$.

Next, we show that $\mathbf{v}=R \mathbf{i}-\mathbf{e}$ is a member of $\mathscr{V}$. Indeed, for every $\mathbf{s} \in \mathscr{K}^{0}$, $\langle\mathbf{v}, \mathbf{s}\rangle=\langle R \mathbf{i}-\mathbf{e}, \mathbf{s}\rangle$, and this is equal to zero according to (13). Moreover, we can invoke Minkowski's inequality and the relation $r_{j} g_{j}=1$ to write

$$
\begin{aligned}
{\left[\sum v_{j}^{2} g_{j}\right]^{1 / 2} } & =\left[\sum\left(r_{j} i_{j}-e_{j}\right)^{2} g_{j}\right]^{1 / 2}=\left[\sum\left(i_{j} r_{j}^{1 / 2}-e_{j} g_{j}^{1 / 2}\right)^{2}\right]^{1 / 2} \\
& \leq\left[\sum i_{j}^{2} r_{j}\right]^{1 / 2}+\left[\sum e_{j}^{2} g_{j}\right]^{1 / 2}
\end{aligned}
$$


The first sum on the right-hand side is finite because $\mathbf{i} \in \mathscr{K}$ according to Theorem 10.2. So too is the second sum because e satisfies Condition E. Thus, $\sum v_{j}^{2} g_{j}<\infty$. So truly, $\mathbf{v} \in \mathscr{V}$.

Finally, we have to show that (15) is satisfied. For any $w \in \mathscr{V}$,

$$
\langle\mathbf{w}, G \mathbf{v}\rangle=\langle\mathbf{w}, G(R \mathbf{i}-\mathbf{e})\rangle=\langle\mathbf{w}, \mathbf{i}-G \mathbf{e}\rangle=\langle\mathbf{w}, \mathbf{i}+\mathbf{h}\rangle,
$$

since $-G \mathbf{e}=\mathbf{h}$ according to the Thevenin-to-Norton conversion of the branch forms. Now $\langle\mathbf{w}, \mathbf{i}\rangle=0$ because of the following facts: $\mathbf{i} \in \mathscr{K} ; \mathscr{K}^{0}$ is dense in $\mathscr{K} ;\langle\mathbf{w}, \mathbf{s}\rangle=0$ for every $\mathbf{s} \in \mathscr{K}^{0}$ by the definition of $\mathscr{V} ; \mathbf{w}$ defines a continuous linear functional on $\mathscr{K}$ because

$$
|\langle\mathbf{w}, \mathbf{s}\rangle| \leq\left|\sum w_{j} g_{j}^{1 / 2} s_{j} r_{j}^{1 / 2}\right| \leq\left[\sum w_{j}^{2} g_{j} \sum s_{j}^{2} r_{j}\right]^{1 / 2}=\|\mathbf{w}\|\|\mathbf{s}\| .
$$

Thus, $\langle\mathbf{w}, G \mathbf{v}\rangle=\langle\mathbf{w}, \mathbf{h}\rangle$, which is (15).

Theorem 13.2. Let $\mathbf{v}$ be dictated by Theorem 12.1 and set $\mathbf{i}=G \mathbf{v}-\mathbf{h}$. Then, i is the member of $\mathscr{K}$ specified by Theorem 10.2 .

Proof. Once again we note that $\mathbf{h}$ satisfies Condition $\mathrm{H}$ if and only if $\mathbf{e}$ satisfies Condition E.

We now show that $\mathbf{i} \in \mathscr{K}$. By using Minkowski's inequality and $i_{j}=g_{j} v_{j}-$ $h_{j}$ as in the preceding proof, we obtain

$$
\left[\sum i_{j}^{2} r_{j}\right]^{1 / 2} \leq\left[\sum v_{j}^{2} g_{j}\right]^{1 / 2}+\left[\sum h_{j}^{2} r_{j}\right]^{1 / 2} .
$$

The right-hand side is finite because $\mathbf{v} \in \mathscr{V}$ and $\mathbf{h}$ satisfies Condition $\mathrm{H}$. Thus, $\mathbf{i} \in \mathscr{I}$.

To complete the proof that $\mathbf{i} \in \mathscr{K}$, we make use of Hilbert's coordinate space $l_{2}$ of one-way infinite vectors of quadratically summable real numbers. Let $\mathscr{K}_{2}$ be the space of all vectors $\left\{r_{j}^{1 / 2} s_{j}\right\}$, where $\mathbf{s}=\left(s_{1}, s_{2}, \ldots\right) \in \mathscr{K}$, and let $\mathscr{V}_{2}$ be the space of all vectors $\left\{g_{j}^{1 / 2} v_{j}\right\}$, where $\mathbf{v}=\left(v_{1}, v_{2}, \ldots\right) \in \mathscr{V}$. Then, $\mathscr{K}_{2}$ and $\mathscr{V}_{2}$ are both subspaces of $l_{2}$. For any $\mathbf{v}_{2} \in \mathscr{V}_{2}$ and any $\mathbf{s}_{2} \in \mathscr{K}_{2}$, we have $\left(\mathbf{v}_{2}, \mathbf{s}_{2}\right)=\sum g_{j}^{1 / 2} v_{j} r_{j}^{1 / 2} s_{j}=\sum v_{j} s_{j}$. By definition, $\mathscr{V}$ is the set of all vectors $\mathbf{v}$ of finite power for which $\sum v_{j} s_{j}=0$ for all $\mathbf{s} \in \mathscr{K}^{0}$, and thereby for all $\mathbf{s} \in \mathscr{K}$ as in the last part of the preceding proof. This shows that $\mathscr{V}_{2}$ and $\mathscr{K}_{2}$ are orthogonal complements of each other; that is, $\mathscr{K}_{2}=\mathscr{V}_{2}{ }^{\perp}$. Let $\mathbf{w}$ be any member of $\mathscr{V}$ and $w_{2}$ its corresponding member of $\mathscr{V}_{2}$. We may write

$$
\langle\mathbf{w}, \mathbf{i}\rangle=\sum g_{j}^{1 / 2} w_{j} r_{j}^{1 / 2} i_{j}=\left(\mathbf{w}_{2}, \mathbf{i}_{2}\right) .
$$

On the other hand, by (15)

$$
\langle\mathbf{w}, \mathbf{i}\rangle=\langle\mathbf{w}, G \mathbf{v}-\mathbf{h}\rangle=0 .
$$

Hence, $\mathbf{i}_{2} \in \mathscr{V}_{2}^{\perp}=\mathscr{K}_{2}$. Therefore, $\mathbf{i} \in \mathscr{K}$.

Finally, we show that (13) is satisfied. Let $s$ be any member of $\mathscr{K}$. Then,

$$
\langle R \mathbf{i}, \mathbf{s}\rangle=\langle R(G \mathbf{v}-\mathbf{h}), s\rangle=\langle\mathbf{v}-R \mathbf{h}, \mathbf{s}\rangle=\langle\mathbf{v}+\mathbf{e}, \mathbf{s}\rangle .
$$

Since $\mathbf{v} \in \mathscr{V},\langle\mathbf{v}, \mathbf{s}\rangle=0$. Thus, $\langle R \mathbf{i}, \mathbf{s}\rangle=\langle\mathbf{e}, \mathbf{s}\rangle$, which is (13). 


\section{NONDISCONNECTABLE 0 -TIPS}

How many 0-tips can a 0-section have? The answer in general is a continuum of them. This is so, for example, for the infinite binary tree of Figure 7. Although each 0-tip therein has an infinity of representatives, it can identified with the unique representative that starts at node $n_{0}$. The cardinality of the set of all one-ended 0 -paths starting at $n_{0}$ is $c$, the cardinality of the continuum, and hence there is a continuum of 0 -tips.

As another example, consider the infinite 0 -graph of Figure 8 . Here too we can restrict our attention to those representatives of 0-tips that start at the 0node $n_{0}$, but now each 0 -tip has an infinity of such representatives. Every one of those representatives can be designated by a sequence $\left\{z_{1}, z_{2}, z_{3}, \ldots\right\}$ of branches, where each $z_{i}$ can be chosen to be either $a_{i}$ or $b_{i}$. There is a continuum of such representatives. Moreover, each 0-tip can be identified as an equivalence class of such sequences, where two sequences are taken to be equivalent if they differ by no more than a finite number of the $z_{i}$. Given any such sequence $S$ we can count all the others in its equivalence class; indeed, count the one that differs from $S$ only in $z_{1}$, then count the two additional ones that differ from $S$ at most only in $z_{1}$ and $z_{2}$, then count the four additional ones that differ from $S$ at most only in $z_{1}, z_{2}$ and $z_{3}$, and so forth. Thus, the cardinality of the set of 0 -tips is $c \div \aleph_{0}=c[13$, p. 299]. Even though this network has the same cardinality of 0 -tips as does that of Figure 7, it can have at most one 0 -tip voltage, whereas the network of Figure 7 can have a continuum of 0 -tip voltages. This is a consequence of a more general result (see Theorem 14.1 below), which we shall now establish.

First however we should explicate what we mean by a " 0 -tip voltage" and by a "node voltage" in a $k$-network. Let $t$ be a 0 -tip, let $x$ be a 1 -node that contains $t$, and let $x_{g}$ be a 0 -node or a 1 -node different from $x$. Then, $x$ 's voltage is

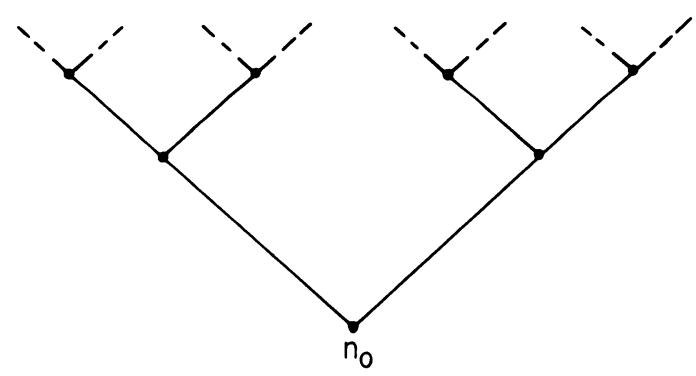

FIGURE 7. The infinite binary tree. It has a continuum of 0 -tips.

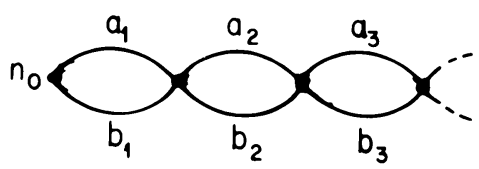

FIGURE 8. A 0 -graph having a continuum of 0 -tips. This is a 1 -times chainlike infinite 0 -graph. 
defined to be the same as $t$ 's voltage, which we now set about defining. As with loops, a path $P$ is said to be perceptible if $\sum_{j \in \Pi} r_{j}<\infty$, where $\Pi$ is the index set for all the branches embraced by $P$.

Definition (perceptible nodes and 0-tips). $x$ and $t$ are said to be perceptible from $x_{g}$ if there exists a perceptible 0-path $P$ that ends at both $x$ and $x_{g}$ and lies in the same 0 -section as the representatives of $t$. $(P$ need not contain a representative of $t$.) Similarly, a 0 -node $n$ is called perceptible from $x_{g}$ if $n$ and $x_{g}$ are connected by a perceptible 0 -path.

Definition (node and 0-tip voltages). Assign a voltage $u_{g}$ to $x_{g}$. Then, $x$ (or $t$ or $n$ ) is said to have a voltage with respect to $x_{g}$ if $x$ (or $t$ or $n$, respectively) is perceptible from $x_{g}$; that voltage is defined to be

$$
u_{g}+\sum_{m \in M} \pm v_{m}
$$

where $M$ is the branch-index set for the branches in a perceptible 0-path $P$ ending at $x_{g}$ and $x$ (or $t$ or $n$, respectively). The $+(-)$ sign is used if the $m$ th branch's orientation agrees (disagrees) with a tracing of $P$ from $x$ to $x_{g}$.

This definition has a meaning because the series converges absolutely, as is shown by the proof of Theorem 11.2. Moreover, $x$ 's voltage is independent of the choice of the path that meets both $x$ and $x_{g}$ so long as the path is perceptible; this follows from Kirchhoff's voltage law which holds for perceptible loops, according to Theorem 11.2 again.

Definition (nondisconnectable 0-tips). Assume that the 0-tips $t_{a}$ and $t_{b}$ possess the representatives $P_{a}$ and $P_{b}$ respectively that meet infinitely often in the following sense: There exists an infinite sequence of 0 -nodes that are all embraced by both $P_{a}$ and $P_{b}$ (perhaps in different orders). Then, $t_{a}$ and $t_{b}$ are called not disconnectable (or nondisconnectable).

This means in effect that the two representatives cannot be isolated from each other by removing a set of branches. Note also that, when $P_{a}$ and $P_{b}$ satisfy the stated conditions, those conditions will also be satisfied by all pairs of representatives for $t_{a}$ and $t_{b}$.

Theorem 14.1. Let the 0-node or 1-node $x_{g}$ of a $k$-network have an assigned voltage. Let $t_{a}$ and $t_{b}$ be two 0-tips which are perceptible from $x_{g}$, have perceptible representatives, and are not disconnectable. Then, they both have voltages $v_{a}$ and $v_{b}$ with respect to $x_{g}$, and moreover $v_{a}=v_{b}$.

Proof. The existence of $v_{a}$ and $v_{b}$ is insured by the perceptibility of $t_{a}$ and $t_{b}$ from $x_{g}$. We can choose any representatives $P_{a}$ and $P_{b}$ for $t_{a}$ and $t_{b}$, and they will be perceptible and will meet infinitely often in the stated sense. Since the representatives of $t_{a}$ and $t_{b}$ lie in the same 0 -section as the perceptible 0 -path $P$ that ends at $x$ and $x_{g}$, all the 0 -nodes of those representatives have voltages with respect to $x_{g}$, and those voltages are determined from $v_{a}$ and $v_{b}$ by formulas such as (16). The absolute convergence of the series in (16) insures that $v_{a}$ (or $v_{b}$ ) is the limit of the voltages at the common 0-nodes shared by $P_{a}$ and $P_{b}$. Thus, $v_{a}=v_{b}$.

A consequence of Theorem 14.1 is that the number of different 0 -tip voltages a 0 -section can have may be radically limited by the structure of the graph. A 


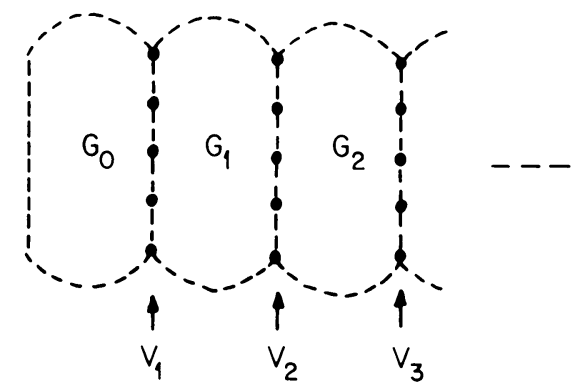

Figure 9. A 5-times chainlike infinite 0-graph. The dots denote the nodes in the various $V_{p}$.

simple example is provided by Figure 8 ; although there is a continuum of 0 -tips in that network, all 0-tip voltages must be the same if they exist at all.

Example. More generally, there can be no more than a finite number $m$ of different 0 -tip voltages in any $m$-times chainlike 0 -graph, a structure examined by Halin [8] and illustrated in Figure 9. Such a structure is the union of an infinity of finite 0-graphs $G_{0}, G_{1}, G_{2}, \ldots$ having the following properties: Each branch appears in exactly one of the $G_{p}$; all 0-nodes have finite degree; $G_{p} \cap G_{p+1}$ is a set $V_{p+1}$ of $m$ 0-nodes; for $|q-p| \geq 2, G_{p} \cap G_{q}$ is void; in each $G_{p}$ with $p \geq 1$, there are $m$ node-distinct finite 0 -paths from the nodes in $V_{p}$ to the nodes in $V_{p+1}$. Thus, a one-way infinite ladder is 2-times chainlike, whereas a two-way infinite ladder is 4-times chainlike.

In general, an $m$-times chainlike 0 -graph can have a continuum of 0-tips. However, one can choose $m$, but no more than $m$, pairwise node-disjoint, one-ended 0 -paths in such a graph. If a voltage-current regime exists according to Theorem 10.2, there can be $m$ (or less) different 0-tip voltages. Any other one-ended 0-path must be nondisconnectable from at least one of the chosen 0paths. Therefore, by Theorem 14.1, there can be no more than $m$ different 0 -tip voltages. In fact, when all $m$ different 0 -tip voltages exist, the 0 -node voltages along any one-ended 0 -path must either converge to one of the $m 0$-tip voltages or oscillate indefinitely.

\section{EFFECTIVELY SHORTED 0-TIPS}

Let us now consider 0-tips that are disconnectable (i.e., not nondisconnectable). Such tips may or may not have voltages in accordance with (16), but, when they do, those voltages are in general different from one another. However, under certain circumstances, the graph and the resistance values of the network may force two such 0-tip voltages to be the same. In effect, the network behaves as though the two tips have been "shorted together".

Example. For the semi-infinite grid of Figure 10, assume that all resistance values vary only in the vertical direction and there are only a finite number of voltage sources, which we take to be in the uppermost branches. Assume also that all 1-nodes are singletons, (i.e., open circuits exist everywhere at infinity). Assign a zero voltage to the infinite 0 -node (i.e., the "ground" node) at the top. If $\sum_{l=0}^{\infty} r_{l}<\infty$, then every 0 -tip with a strictly vertical representative has a 


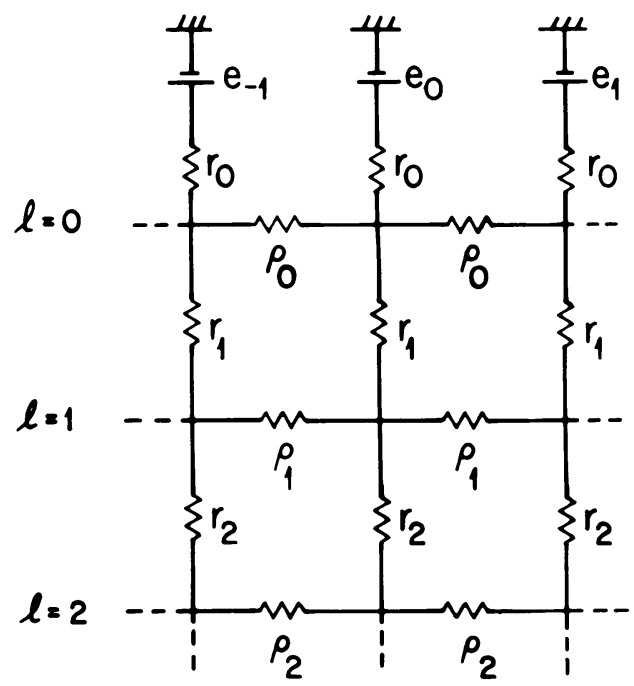

FIGURE 10. A semi-infinite grid. The $e_{m}$ denote voltage sources, and the $r_{l}$ and $\rho_{l}$ denote positive resistances.

voltage, which is the limit of the node voltages along that representative. If in addition every horizontal resistance $\rho_{l}$ is $1 \mathrm{ohm}$, then those vertically downward 0 -tips must have the same voltage, as we shall show below. On the other hand, if the horizontal resistances increase rapidly enough to yield $\sum_{l=0}^{\infty} \rho_{l}^{-1}<\infty$, then the vertically downward 0 -tips can have different voltages [27].

Some conditions that force two disconnectable 0 -tips to have the same voltage are the following.

Theorem 15.1. Let two 0-tips $t_{a}$ and $t_{b}$ be perceptible from a node $x_{g}$ with a given voltage. Let $\left\{y_{a n}\right\}_{n=1}^{\infty}$ (and $\left.\left\{y_{b n}\right\}_{n=1}^{\infty}\right)$ be an infinite sequence of 0 nodes embraced by a perceptible representative $P_{a}$ of $t_{a}$ (respectively, $P_{b}$ of $\left.t_{b}\right)$. Assume that, for each $n, y_{a n}$ and $y_{b n}$ are connected by a 0-path $Q_{n}$ that terminates at $y_{a n}$ and $y_{b n}$. Let $J_{n}$ denote the branch index set for $Q_{n}$ and assume that $\min J_{n}=\min \left\{j: j \in J_{n}\right\} \rightarrow \infty$ as $n \rightarrow \infty$. Assume furthermore that

$$
\sum_{j \in J_{n}} r_{j}<M,
$$

where $M$ is a constant independent of $n$. Then, $t_{a}$ and $t_{b}$ have the same voltage with respect to $x_{g}$.

Proof. We proceed as in the proof of Theorem 14.1, but now the voltage at $y_{a n}$ need not be the same as the voltage at $y_{b n}$. They differ by

$$
\sum_{j \in J_{n}} \pm\left(e_{j}-i_{j} r_{j}\right) .
$$

The $+(-)$ sign is chosen if that branch's orientation agrees (disagrees) with a tracing from $y_{a n}$ to $y_{b n}$ along $Q_{n}$. By virtue of the proof of Theorem 14.1, all we need to show is that (18) tends to zero as $n \rightarrow \infty$. 
Let $\sum_{n}$ denote $\sum_{j \in J_{n}}$. Then

$$
\sum_{n}\left|i_{j} r_{j}\right| \leq \sum_{n}\left|i_{j}\right| r_{j}^{1 / 2} r_{j}^{1 / 2} \leq\left[\sum_{n} i_{j}^{2} r_{j} \sum_{n} r_{j}\right]^{1 / 2} .
$$

But, $\sum_{j=0}^{\infty} i_{j}^{2} r_{j}<\infty$ since $\mathbf{i} \in \mathscr{K}$ by Theorem 10.2. Since $\min J_{n} \rightarrow \infty$ as $n \rightarrow \infty$, it follows that $\sum_{n} i_{j}^{2} r_{j} \rightarrow 0$ as $n \rightarrow \infty$. In view of (17), we can conclude that $\sum_{n}\left|i_{j} r_{j}\right| \rightarrow 0$.

Similarly, upon setting $g_{j}=r_{j}^{-1}$, we may write

$$
\sum_{n}\left|e_{j}\right|=\sum_{n}\left|e_{j}\right| g_{j}^{1 / 2} r_{j}^{1 / 2} \leq\left[\sum_{n} e_{j}^{2} g_{j} \sum_{n} r_{j}\right]^{1 / 2} .
$$

By Condition E, $\sum_{j=0}^{\infty} e_{j}^{2} g_{j}<\infty$. So, as $n \rightarrow \infty, \sum_{n} e_{j}^{2} g_{j} \rightarrow \infty$ because $\min J_{n} \rightarrow \infty$. Again by (17), $\sum_{n}\left|e_{j}\right| \rightarrow 0$.

Combining these two results, we have it that (18) tends to zero as $n \rightarrow \infty$.

\section{THOMSON'S LEAST POWER PRINCIPLE}

Another approach to the theory of finite networks is to identify the voltagecurrent regime as that regime for which the power dissipated is minimized [ 9 , p. 322]. The same property holds for $k$-networks.

Let us assume that all the branches are in their Thevenin form (Figure 4). Let $\mathscr{L}_{e}$ be the subset of $\mathscr{K}$ defined by

$$
\mathscr{L}_{e}=\{\mathbf{s} \in \mathscr{K}:\langle\mathbf{e}, \mathbf{s}\rangle=\langle\mathbf{e}, \mathbf{i}\rangle\},
$$

where as always $\mathbf{i}$ is the current vector dictated by Theorem 10.2.

Theorem 16.1. Under the hypothesis of Theorem 10.2, $\mathbf{i}$ is that member $\mathbf{s} \in \mathscr{L}_{e}$ for which $\sum s_{j}^{2} r_{j}$ is a minimum. There is only one such member of $\mathscr{L}_{\boldsymbol{e}}$.

Proof. Set $\mathbf{s}=\mathbf{i}+\Delta \mathbf{i}$. So, $\Delta \mathbf{i} \in \mathscr{K}$. Moreover,

$$
\langle\mathbf{e}, \mathbf{s}\rangle=\langle\mathbf{e}, \mathbf{i}\rangle+\langle\mathbf{e}, \Delta \mathbf{i}\rangle .
$$

Since $\mathbf{s} \in \mathscr{L}_{e}$,

$$
\langle\mathbf{e}, \Delta \mathbf{i}\rangle=0 .
$$

We shall show that

$$
\langle R(\mathbf{i}+\Delta \mathbf{i}), \mathbf{i}+\Delta \mathbf{i}\rangle-\langle R \mathbf{i}, \mathbf{i}\rangle
$$

is a positive quantity. Indeed, (20) is equal to

$$
\langle R \mathbf{i}, \Delta \mathbf{i}\rangle+\langle R \Delta \mathbf{i}, \mathbf{i}\rangle+\langle R \Delta \mathbf{i}, \Delta \mathbf{i}\rangle=2\langle R \mathbf{i}, \Delta \mathbf{i}\rangle+\langle R \Delta \mathbf{i}, \Delta \mathbf{i}\rangle .
$$

By (13), $\langle R \mathbf{i}, \Delta \mathbf{i}\rangle=\langle\mathbf{e}, \Delta \mathbf{i}\rangle$, which by (19) is zero. Hence, (20) equals $\langle R \Delta \mathbf{i}, \Delta \mathbf{i}\rangle$ $=\sum r_{j}\left(\Delta i_{j}\right)^{2}$. This is strictly positive whenever $\Delta \mathbf{i} \neq 0$.

Now, let

$$
\mathscr{T}_{e}=\left\{\mathbf{s} \in \mathscr{K}: s_{j}=i_{j} \text { whenever } e_{j} \neq 0\right\} .
$$

$\mathscr{T}_{e}$ is a subset of $\mathscr{L}_{e}$, and therefore we have 
Corollary 16.2. For any $\mathbf{s} \in \mathscr{T}_{e}, \sum s_{j}^{2} r_{j} \geq \sum i_{j}^{2} r_{j}$, with strict inequality occurring if $\mathbf{s} \neq \mathbf{i}$.

A way of understanding this corollary is to turn our attention to the reduced network induced by those branches that do not possess voltage sources. The currents in the branches with nonzero voltage sources can be taken to be a set of given current sources feeding the said reduced network. According to the corollary, the currents are distributed throughout the reduced network to minimize the power dissipated therein.

In the dual case the following results can be established in just the same way. Assume that all the branches are in the Norton form (Figure 6) and let

$$
\mathscr{M}_{h}=\{\mathbf{w} \in \mathscr{V}:\langle\mathbf{w}, \mathbf{h}\rangle=\langle\mathbf{v}, \mathbf{h}\rangle\} .
$$

Theorem 16.3. If $\mathbf{v}$ is the voltage vector specified by Theorem 12.1 , then $\mathbf{v}$ is that member of $\mathscr{M}_{h}$ for which $\sum w_{j}^{2} g_{j}$ is a minimum. There is only one such member of $\mathscr{M}_{h}$.

Now, let $J$ be the index set for all the branches having nonzero current sources. Let

$$
\mathscr{N}_{h}=\left\{\mathbf{w} \in \mathscr{V}: w_{j}=v_{j} \text { whenever } j \in J\right\} .
$$

Clearly, $\mathscr{N}_{h}$ is a subset of $\mathscr{M}_{h}$. Thus, we have

Corollary 16.4. If $\mathbf{v}$ is given by Theorem 12.1 and if $\mathbf{w} \in \mathscr{N}_{h}$, then $\sum w_{j}^{2} g_{j} \geq$ $\sum v_{j}^{2} g_{j}$, with strict inequality holding if $\mathbf{w} \neq \mathbf{v}$.

\section{RESISTANCE THEOREMS}

Another way to examine the behavior of a purely resistive (i.e., no sources) $k$-network is to observe its driving-point resistance $R_{D}$ between a pair of nodes $x_{1}^{p}$ and $x_{2}^{q}$. This is illustrated in Figure 11. The ranks $p$ and $q$ need not be the same. An additional branch $b_{0}$ with voltage source $e_{0}$ and resistance $r_{0}$ is connected to $x_{1}^{p}$ and $x_{2}^{q}$ by having the 0-nodes of $b_{0}$ embraced by $x_{1}^{p}$ and $x_{2}^{q}$, one to each. Then, $R_{D}=v_{0} / i_{0}=R_{0}-r_{0}$, where $R_{0}=e_{0} / i_{0}$ is the driving-point resistance as seen from $e_{0}$. Now, $R_{0}$ (or $R_{D}$ ) is a function of all the branch resistances $r_{j}$ including $r_{0}$ (respectively, excluding $r_{0}$ ). We shall prove in this section that $R_{0}$ and $R_{D}$ are concave-downward functions of those resistances; that is, with $r_{j}$ and $r_{j}^{\prime}$ denoting positive resistances, we have

$$
\begin{aligned}
& R_{0}\left(r_{0}+r_{0}^{\prime}, r_{1}+r_{1}^{\prime}, \ldots\right) \geq R_{0}\left(r_{0}, r_{1}, \ldots\right)+R_{0}\left(r_{0}^{\prime}, r_{1}^{\prime}, \ldots\right), \\
& R_{D}\left(r_{1}+r_{1}^{\prime}, r_{2}+r_{2}^{\prime}, \ldots\right) \geq R_{D}\left(r_{1}, r_{2}, \ldots\right)+R_{D}\left(r_{1}^{\prime}, r_{2}^{\prime}, \ldots\right) .
\end{aligned}
$$

This will extend a result of Shannon and Hagelbarger [11, 15] to our $k$-networks.

Let $\mathscr{K}$ now be the space of allowable branch-current vectors for the network obtained by augmenting the $k$-network with the branch $b_{0}$, as indicated in Figure 11. We will need the condition that $i_{0} \neq 0$ whenever $e_{0} \neq 0$.

Lemma 17.1. Assume that $e_{0} \neq 0$ and that $e_{j}=0$ for $j=1,2, \ldots$ Then, for the current vector $\mathbf{i}=\left(i_{0}, i_{1}, \ldots\right)$ dictated by Theorem 10.2 , we have $i_{0} \neq 0$ if and only if $\mathscr{K}$ contains at least one current vector $\mathbf{f}=\left(f_{0}, f_{1}, f_{2}, \ldots\right)$ with $f_{0} \neq 0$.

Proof. Only if. This is obvious. 


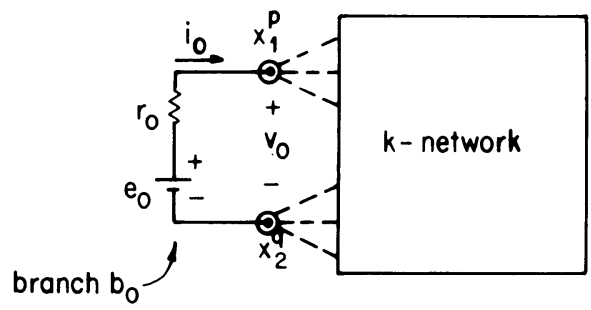

FIGURE 11. The set-up for measuring the driving-point resistance $R_{D}=e_{0} / i_{0}-r_{0}$ between the two nodes $x_{1}^{p}$ and $x_{2}^{q}$ of a purely resistive $k$-network. The $k$-network has no sources.

If. Suppose $\mathscr{K}$ possesses such an $\mathbf{f}$ and yet $i_{0}=0$. Then, $e_{0} i_{0}=0$. Set $\mathbf{s}=\mathbf{i}$ in (13). This yields $e_{0} i_{0}=\sum i_{j}^{2} r_{j}$. Hence, $i_{j}=0$ for every $j$. Thus, by (13) with $\mathbf{s}=\mathbf{f}$, we have $e_{0} f_{0}=\sum r_{j} i_{j} f_{j}=0$. Therefore, $e_{0}=0$; this contradicts the hypothesis that $e \neq 0$.

Theorem 17.2. Let $R_{0}=e_{0} / i_{0}$ be measured in accordance with Figure 11 where $e_{0} \neq 0$, the k-network has no sources, and the current regime is dictated by Theorem 10.2. If $\mathscr{K}$ contains at least one current vector $\mathbf{f}=\left(f_{0}, f_{1}, \ldots\right)$ with $f_{0} \neq 0$, then $R_{0}=e_{0} / i_{0}$ is a concave downward function of the $r_{j}$, that is, (21) holds for all positive $r_{j}$ and $r_{j}^{\prime}$.

Proof. Choose any real nonzero number $I_{0}$ and fix it. Since $i_{0} \neq 0$ by Lemma 17.2, we can always adjust $e_{0}$ to make $i_{0}=I_{0}$; indeed, just multiply $\mathbf{e}$ and $\mathbf{i}$ in (13) by $I_{0} / i_{0}$.

Next, choose any collection of positive resistances $r_{j}$ and $r_{j}^{\prime}$ for all $j=$ $0,1,2, \ldots$. Consider three cases where in each case $e_{0}$ has been adjusted to make $i_{0}=I_{0}$. In the first case, the branch resistances are $r_{j}+r_{j}^{\prime}$, the adjusted value of $e_{0}$ is denoted by $E_{0}$, the branch currents are $I_{j}$, and

$$
R_{0}\left(r_{0}+r_{0}^{\prime}, r_{1}+r_{1}^{\prime}, \ldots\right)=\frac{E_{0}}{I_{0}}=\sum\left(r_{j}+r_{j}^{\prime}\right) \frac{I_{j}^{2}}{I_{0}^{2}} .
$$

The last equation is obtained by setting $\mathbf{s}=\mathbf{I}=\left(I_{0}, I_{1}, \ldots\right)$ in (13) and then dividing by $I_{0}^{2}$. In the second and third cases, the branch resistances are $r_{j}$ and respectively $r_{j}^{\prime}$, the adjusted values of $e_{0}$ are denoted by $e_{0}$ and $e_{0}^{\prime}$, the branch currents are $i_{j}$ and $i_{j}^{\prime}$, and

$$
R_{0}\left(r_{0}, r_{1}, \ldots\right)=\frac{e_{0}}{I_{0}}=\sum r_{j} \frac{i_{j}^{2}}{I_{0}^{2}}
$$

respectively

$$
R_{0}\left(r_{0}^{\prime}, r_{1}^{\prime}, \ldots\right)=\frac{e_{0}^{\prime}}{I_{0}}=\sum r_{j}^{\prime} \frac{i_{j}^{\prime 2}}{I_{0}^{2}}
$$

For the second case, $\mathbf{I}$ is not in general equal to the solution $\mathbf{i}$ but is a member of $\mathscr{T}_{e}$ (see $\left.\S 16\right)$. So, by Corollary $16.2, \sum r_{j} I_{j}^{2} \geq \sum r_{j} i_{j}^{2}$. In the same way we 
get $\sum r_{j}^{\prime} I_{j}^{2} \geq \sum r_{j}^{\prime} i_{j}^{\prime 2}$. Hence,

$$
\begin{aligned}
& R_{0}\left(r_{0}+r_{0}^{\prime}, r_{1}+r_{1}^{\prime}, \ldots\right)=\sum r_{j} \frac{I_{j}^{2}}{I_{0}^{2}}+\sum r_{j}^{\prime} \frac{I_{j}^{2}}{I_{0}^{2}} \\
& \quad \geq \sum r_{j} \frac{i_{j}^{2}}{I_{0}^{2}}+\sum r_{j}^{\prime} \frac{i_{j}^{\prime 2}}{I_{0}^{2}}=R_{0}\left(r_{0}, r_{1}, \ldots\right)+R_{0}\left(r_{0}^{\prime}, r_{1}^{\prime}, \ldots\right),
\end{aligned}
$$

as asserted.

With regard to $R_{D}=v_{0} / i_{0}$, we need merely subtract $r_{0}$ from $R_{0}$ to obtain Corollary 17.3. Under the hypothesis of Theorem 17.2, (22) holds.

Note that, since we are dealing with a $k$-network, the nodes $x_{1}^{p}$ and $x_{2}^{q}$ in Figure 11 may be effectively shorted together by the network. Thus, a pure voltage source of value $v_{0}$ applied to $x_{1}^{p}$ and $x_{2}^{q}$ may yield an infinite $i_{0}$. This is why our result on $R_{D}$ had to be derived from $R_{0}$ rather than directly.

The concavity property continues to hold even when some of the $r_{j}^{\prime}$ are zero. The same proof holds except that in the third case we would be dealing with a network some of whose branches have zero resistance. Instead of this, we will short together the nodes of any such branch to make it into a self-loop; in the resulting network those self-loop branches, none of which have sources, will carry zero currents when $e_{0}$ is applied as in Figure 11. Thus, we can still invoke Corollary 16.2 for the latter network to write $\sum r_{j}^{\prime} I_{j}^{2} \geq \sum r_{j}^{\prime} i_{j}^{\prime 2}$, as needed.

Corollary 17.4. Theorem 17.2 continues to hold even when some of the $r_{j}^{\prime}$ are equal to zero.

Another corollary is Rayleigh's monotonicity law. For $k$-networks we have

Corollary 17.5. Assign two, possibly different, resistance values $r_{j}$ and $r_{j}^{\prime}$ to each branch of the $k$-network of Figure 11 such that $r_{0}=r_{0}^{\prime}$ and $r_{j} \geq r_{j}^{\prime}$ for $j=1,2, \ldots$. Then,

$$
R_{0}\left(r_{0}, r_{1}, r_{2}, \ldots\right) \geq R_{0}\left(r_{0}, r_{1}^{\prime}, r_{2}^{\prime}, \ldots\right)
$$

and

$$
R_{D}\left(r_{1}, r_{2}, \ldots\right) \geq R_{D}\left(r_{1}^{\prime}, r_{2}^{\prime}, \ldots\right) .
$$

These results also hold in the dual approach, where now the $R$ 's and $r$ 's are replaced by $G$ 's and $g$ 's.

\section{SOME FINAL REMARKS}

1. Pure sources. We have assumed that every branch has a positive (not zero) branch resistance. However, we can allow some branches to have zero resistance, that is, to be pure voltage sources; in fact, pure current sources can also be allowed. Our theory can be so extended by using the technique of transferring pure sources into branches with positive resistances and adapting the arguments employed in $\S \S$ VII, VIII, and XII of [24].

2. Reciprocity theorem. The reciprocity theorem continues to hold for $k$-networks. That theorem states that the current in branch $j$ due to a unit voltage source in branch $m$ is equal to the current in branch $m$ due to a unit voltage source in branch $j$. Flanders' proof of this fact (see Corollary 3 in [7]) extends directly to our $k$-networks. 
3. Transfinite digraphs. Some manifest alterations in our definitions lead to transfinite digraphs. Let us just sketch the construction. We now start with an ordinary digraph, that is, a pair $\left(\mathscr{A}, \mathscr{V}^{0}\right)$, where $\mathscr{A}$ is the set of arcs and $\mathscr{V}^{0}$ is the set of vertices-now called 0-vertices. We then partition all one-ended dipaths into equivalence classes by treating two dipaths as being equivalent if they differ at most by a finite number of 0 -vertices and arcs. Such an equivalence class can be called a 0 -ditip. A 0 -ditip is either a 0 -intip or a 0 -outtip if any one of its representatives is directed toward or respectively away from the 0 -ditip. Then, a 1-vertex is a set of 0-ditips plus possibly one 0 -vertex, and it is required that every 0 -ditip be a member of some 1 -vertex and that any two 1-vertices have a void intersection. Finally, a 1-digraph is the triplet $\left(\mathscr{A}, \mathscr{V}^{0}, \mathscr{V}^{1}\right)$, where $\mathscr{V}^{1}$ is the set of 1 -vertices.

This construction can be continued to obtain transfinite digraphs of higher ranks, namely, $k$-digraphs. Corresponding to each $k$-digraph there is an underlying $k$-graph, which can be partitioned into $q$-sections with $0 \leq q \leq k$ as above. Now however, we can define a strong $q$-section as a reduced digraph induced by a maximal set of arcs that are strongly $q$-connected. Each strong $q$-section will be entirely contained within a $q$-section.

\section{REFERENCES}

1. L. De Michele and P. M. Soardi, $A$ Thomson's principle for infinite, nonlinear, resistive networks, Proc. Amer. Math. Soc. 109 (1992), 461-468.

2. V. Dolezal, Nonlinear networks, Elsevier, New York, 1977.

3. __, Monotone operators and applications in control and network theory, Elsevier, New York, 1979.

4. V. Dolezal and A. H. Zemanian, Hilbert networks II: Some qualitative properties, SIAM J. Control 13 (1975), 153-161.

5. P. G. Doyle, Electric currents in infinite networks, preprint, 1988.

6. P. G. Doyle and J. L. Snell, Random walks and electric networks, The Carus Mathematical Monographs, Math. Assoc. Amer., Washington, D.C., 1984.

7. H. Flanders, Infinite networks: I-Resistance networks, IEEE Trans. Circuit Theory CT-18 (1971), 326-331.

8. R. Halin, Some path problems in graph theory, Abh. Math. Sem. Univ. Hamburg 44 (1975), 175-186.

9. J. Jeans, Electricity and magnetism, 5th ed., Cambridge Univ. Press, London, 1927.

10. L. A. Liusternik and V. J. Sobolev, Elements of functional analysis, Ungar, New York, 1961.

11. H. M. Melvin, On the concavity of resistance functions, J. Appl. Phys. 27 (1956), 658-659.

12. C. St. J. A. Nash-Williams, Random walks and electric currents in networks, Proc. Cambridge Philos. Soc. 55 (1959), 181-194.

13. J. E. Rubin, Set theory, Holden-Day, San Francisco, Calif., 1967.

14. E. Schlesinger, Infinite networks and Markov chains, preprint, 1989.

15. C. E. Shannon and D. W. Hagelbarger, Concavity of resistance functions, J. Appl. Phys. 27 (1956), 42-43.

16. F. Spitzer, Principles of random walk, Van Nostrand, Princeton, N.J., 1964.

17. P. M. Soardi and W. Woess, Uniqueness of currents in infinite resistive networks, Discrete Appl. Math. 31 (1991), 37-49.

18. C. Thomassen, Resistances and currents in infinite electrical networks, J. Combin. Theory Ser. B 49 (1990), 87-102.

19. A. H. Zemanian, Countably infinite networks that need not be locally finite, IEEE Trans. Circuits and Systems CAS-21 (1974), 274-277. 
20. __ Infinite networks of positive operators, Circuit Theory and Applications 2 (1974), 69-78.

21. _ Connections at infinity of a countable resistive network, Circuit Theory and Applications 3 (1975), 333-337.

22. __ Infinite electrical networks, Proc. IEEE 64 (1976), 6-17.

23. _ The limb analysis of countably infinite electrical networks, J. Combin. Theory Ser. B 24 (1978), 76-93.

24. __ Infinite electrical networks with finite sources at infinity, IEEE Trans. Circuits and Systems CAS-34 (1987), 1518-1534.

25. __ Infinite electrical networks: A reprise, IEEE Trans. Circuits and Systems CAS-35 (1988), 1346-1358.

26. __ Transfinite cascades, IEEE Trans. Circuits and Systems 38 (1991), 78-85.

27. _. Boundary conditions at infinity for a discrete form of $\nabla \cdot(\sigma \nabla \Phi)=\alpha \Phi, \alpha \geq 0$, State University of New York at Stony Brook, CEAS Technical Report 523, August, 1988.

28. __ Transfinite random walks based on electrical networks, State University of New York at Stony Brook, CEAS Tech. Rep. 604, June 4, 1991.

29. _ Random walks on $\omega$-networks, Harmonic Analysis and Discrete Potential Theory (M. Picardello, Editor), Plenum, London, 1992 (in press).

Department of Electrical Engineering, State University of New York at Stony Brook, Stony Brook, New York 11794-2350

E-mail address: zeman@sbee.sunysb.edu 